\title{
Pseudodifferential Operators on Stratified Manifolds
}

\author{
V. E. Nazaikinskii, A. Yu. Savin, and B. Yu. Sternin
}

\section{Contents}

Introduction 2

\begin{tabular}{|lll}
1 & Generalized elliptic operators & 3 \\
\hline
\end{tabular}

1.1 Local operators with parameter . . . . . . . . . . . . . . 3

1.2 Ellipticity and the Fredholm propertv . . . . . . . . . . . . . 3

2 Localization 4

2.1 Localizing classed . . . . . . . . . . . . . . . . . . . 4

2.2 Conditions on the action of $C(X) \ldots \ldots \ldots \ldots \ldots$

2.3 Localization in the algebra $\mathcal{A} \ldots \ldots \ldots \ldots \ldots$

2.4 Properties of local representatives . . . . . . . . . . . . . . . 6

$\begin{array}{llr}3 & \text { Cones and infinitesimal operators } & 9\end{array}$

3.1 Operators on the cond . . . . . . . . . . . . . . . . . . . . 10

3.2 Infinitesimal operators . . . . . . . . . . . . . . . . . . . 11

3.3 The decomposition problem for infinitesimal operators . . . . . . . . . 13

3.4 Svmbols in the small . . . . . . . . . . . . . . . . . . . . . . . 13

$\begin{array}{lll}4 \text { Stratified manifolds } & 19\end{array}$

4.1 Definition of stratified manifolds . . . . . . . . . . . . . . . . . . . . 19

4.2 Generating vector fields of families of diffeomorphisms . . . . . . . . . 21

4.3 The blow-un . . . . . . . . . . . . . . . . . . . . . . . . 21

4.4 The cone bundle . . . . . . . . . . . . . . . . . . . . . . . . . 22

4.5 Measures on a stratified manifold $\ldots \ldots \ldots \ldots \ldots \ldots$

4.6 The cotangent bundle . . . . . . . . . . . . . . . . . . . . . . . . 23

4.7 The space $C^{\infty}(\mathcal{M}) \quad \ldots \ldots \ldots \ldots$

$\begin{array}{lll}5 & \text { Pseudodifferential operators } & 24\end{array}$

5.1 Negligible operators . . . . . . . . . . . . . . . . . . . . . . 25

5.2 Definition of pseudodifferential operators . . . . . . . . . . . 26

5.3 The simplest properties of pseudodifferential operators $\ldots \ldots \ldots . .27$

5.4 Invariance with respect to changes of variables . . . . . . . . . . . . . . . 29 
$\begin{array}{lll}6 & \text { Svmbols and quantization } & 30\end{array}$

6.1 Infinitesimal operators and svmbols of pseudodifferential operators . . . . . 30

6.2 Conditions for the Fredholm property of svmbols . . . . . . . . . . . . . . 31

6.3 Compatibilitv conditions and quantization . . . . . . . . . . . . . . 31

6.4 Main properties of pseudodifferential operators . . . . . . . . . . . . . . . 33

6.5 Ellipticity and the Fredholm property . . . . . . . . . . . . . . . . . . . . . 33

7 Proof of Proposition 16

$\begin{array}{ll}\text { Bibliography } & 35\end{array}$

\section{Introduction}

Pseudodifferential operators ( $\Psi D O)$ are an important tool in elliptic theory on manifolds with singularities. When studying "topological" issues of this theory, including the homotopy classification and index theory of elliptic operators, the main question of interest in $\Psi D O$ theory is the structure of the algebra of principal symbols (which determine the Fredholm property of an operator) and the relationship between this algebra and the algebra of operators themselves, in particular, the compatibility conditions for the principal symbol components corresponding to various strata of the manifold and the existence of a quantization procedure (i.e., the construction of an operator from its principal symbol). More subtle issues related to the so-called complete symbols of $\Psi D O$ and specific analytic formulas describing $\Psi D O$ do not play any significant role in this field.

In this connection, it is of interest to devise a $\Psi D O$ theory in which the principal symbol plays the main role and lower-order terms are touched only when this is absolutely necessary. Such a construction is given in the present paper.

We consider a specific class of manifolds with singularities, namely, stratified manifolds, and describe a class of $\Psi D O$ related to differential operators with degeneration of first-order with respect to the distance to the strata on such manifolds. (For manifolds with isolated singularities, this is a Fuchs type degeneration.)

We restrict ourselves to the case of zero-order $\Psi D O$ in $L^{2}$ spaces, which is of main interest here. (Operators of nonzero order can be reduced to zero-order operators by the order reduction procedure.) The definition of $\Psi D O$ adopted in our approach uses induction over the number of strata in the manifold. The induction process inevitably involves $\Psi D O$ with parameters; hence we state all definitions for $\Psi D O$ with parameters from the very beginning. This simplifies the argument greatly and does not lead to any serious complications in the statements themselves.

Note that $\Psi \mathrm{DO}$ on manifolds with singularities were considered by numerous authors. For example, $\Psi$ DO with cone and edge degeneration in so-called edge Sobolev spaces were considered in $[8,16]$; the methods of these papers were developed for more complicated singularities in [5]; a close class of $\Psi \mathrm{DO}$ on manifolds with corners was studied in [10]. Groupoids were used in [12] to analyze $\Psi D O$ on manifolds with singularities, and the paper [15] uses localization methods in $C^{*}$-algebras for the same purpose.

In this paper, we first develop some auxiliary results pertaining to the localization principle for abstract local operators (concerning this principle, see also $[2,9,15,17-19]$ ). 
Then we consider $\Psi$ DO.

Some results were partly announced without proof in our paper [11] written jointly with B.-W. Schulze.

\section{Generalized elliptic operators}

Let $H$ be a Hilbert space. By $\mathcal{B} H$ and $\mathcal{K} H$ we denote the algebra of bounded linear operators in $H$ and the ideal of compact operators, respectively. Suppose that $H$ is equipped with the structure of a unital $*$-module over the $C^{*}$-algebra $C(X)$ of continuous functions on a compact set $X$. An operator $A \in \mathcal{B} H$ is called a generalized elliptic operator in the sense of Atiyah [4] if it is Fredholm and local, i.e., compactly commutes with the action of $C(X)$. We need a natural generalization of these notions to the case of operator families.

\subsection{Local operators with parameter}

Let $Y$ be a Hausdorff locally compact topological space, in general, noncompact (which will play the role of a parameter space). The $C^{*}$-algebra $C(Y, \mathcal{B} H)$ of bounded continuous operator families $A: Y \longrightarrow \mathcal{B} H$ with the sup-norm

$$
\|A\|=\sup _{y \in Y}\|A(y)\|
$$

contains the closed $*$-ideal

$$
\mathcal{J}=C_{0}(Y, \mathcal{K} H)
$$

of compact-valued families tending to zero at infinity in norm. By $\mathcal{A}$ we denote the $C^{*}$-subalgebra of $C(Y, \mathcal{B} H)$ consisting of families $A(y)$ such that

$$
[A(y), \varphi] \in \mathcal{J} \quad \text { for any function } \varphi \in C(X) .
$$

The elements of $\mathcal{A}$ are called local operators with a parameter.

\subsection{Ellipticity and the Fredholm property}

The Fredholm property can naturally be generalized to the case of operator families as follows. A family $A \in C(Y, \mathcal{B} H)$ is said to be Fredholm with parameter $y$ if the operator $A(y)$ is Fredholm for all $y$ and invertible for large $y$ (i.e., outside some subset in $Y$ ) and the inverse $A^{-1}(y)$ is bounded uniformly with respect to $y$ for large $y$.

Now we can give the notion of generalized ellipticity in the sense of Atiyah. An operator family $A \in C(Y, \mathcal{B} H)$ is called a generalized elliptic operator with a parameter if it is local (i.e., $A \in \mathcal{A}$ ) and Fredholm with parameter in the sense described above.

Let

$$
\sigma: \mathcal{A} \longrightarrow \mathcal{A} / \mathcal{J}
$$

be the natural projection onto the quotient algebra. One can readily verify that a necessary and sufficient condition that an operator family $A \in \mathcal{A}$ is elliptic with a parameter is 
that the corresponding element $\sigma(A)$ of the quotient algebra is invertible. (As usual, when proving that the family is invertible for large parameter values, one applies the Neumann series.) Hence this condition can be used as an (equivalent) definition of ellipticity:

Definition 1. The element $\sigma(A)$ is called the symbol of a local operator $A \in \mathcal{A}$ with a parameter. The operator $A \in \mathcal{A}$ is said to be elliptic with a parameter if its symbol $\sigma(A)$ is invertible.

The equivalence of the two definitions is none other than the finiteness theorem.

Theorem 2. If a family $A \in \mathcal{A}$ is elliptic with a parameter, then it is Fredholm with a parameter. The converse is also true.

Thus the main analytical task of elliptic theory of operators with a parameter (just as in the parameter-free case) is the study of symbols $\sigma(A)$ and the structure of the symbol algebra $\mathcal{A} / \mathcal{J}$; in particular, it is of interest to find specific subalgebras of $\mathcal{A}$ for which the symbols can be described constructively.

\section{Localization}

However, it turns out that under certain additional conditions there are some nontrivial assertions even in elliptic theory for the entire algebra $\mathcal{A}$. Namely, the invertibility of an element $\sigma(A) \in \mathcal{A} / \mathcal{J}$ is reduced to that of a set $\left\{p_{x}(A)\right\}$ of "local representatives" labelled by points $x \in X$.

\subsection{Localizing classes}

Throughout the following, we assume that $X$ is a Hausdorff compactum. For an arbitrary point $x \in X$, consider the set $\mathcal{F}_{x} \subset C(X)$ of functions $\varphi(y)$ such that $0 \leq \varphi(y) \leq 1$ and $\varphi(y)=1$ in some (depending on $\varphi$ ) neighborhood of $x$. This set is bounded (the norm of each element is equal to 1) and multiplicative. It follows from the Urysohn lemma that the ordering

$$
\varphi \prec \psi \Longleftrightarrow \varphi \psi=\psi
$$

makes it a directed set, which will be called the localizing class at $x$ (cf. [9, §5.1]). The supports of its elements "shrink" to $x$.

\subsection{Conditions on the action of $C(X)$}

We assume that the following two conditions are satisfied.

$1^{\circ}$. The representation of $C(X)$ in $\mathcal{B H}$ is faithful (i.e., $C(X)$ is embedded in $\mathcal{B} H$ as a subalgebra), and $C(X) \cap \mathcal{K} H=\{0\}$.

$2^{\circ}$. For each $x \in X$, the localizing class $\mathcal{F}_{x}$ strongly converges to zero in $\mathcal{B} H$,

$$
\mathrm{s}-\lim _{\varphi \in \mathcal{F}_{x}} \varphi=0 .
$$

In other words, $H$ does not contain elements "concentrated at $x$." (If $H=L^{2}(X, d \mu)$, then condition $2^{\circ}$ means that the measure $\mu$ is purely nonatomic.) 


\subsection{Localization in the algebra $\mathcal{A}$}

We construct local representatives of elements $A \in \mathcal{A}$ on the basis of the localization principle in $C^{*}$-algebras, which we use in the form given in [15, Proposition 3.1].

The algebra $C(X)$ is naturally embedded in $\mathcal{A}$ as a commutative subalgebra. (Its elements are constant functions of the parameter $y$ ). Let $\mathcal{I}_{x} \subset C(X)$ be the maximal ideal of functions vanishing at $x \in X$, and let $\mathcal{J}_{x}$ be the ideal generated by $\mathcal{I}_{x}$ in $\mathcal{A}$. The quotient algebra $\mathcal{A}_{x}=\mathcal{A} / \mathcal{J}_{x}$ is called the local algebra (at $x$ ), and the coset $p_{x}(A) \in \mathcal{A}_{x}$ of an element $A \in \mathcal{A}$ is called the local representative of $A$ at $x$. Here

$$
p_{x}: \mathcal{A} \longrightarrow \mathcal{A}_{x}
$$

is the natural projection.

Theorem 3. (a) The $C^{*}$-algebra homomorphism

$$
\left(\prod_{x \in X} p_{x}\right): \mathcal{A} \longrightarrow \prod_{x \in X} \mathcal{A}_{x}
$$

induces a well-defined homomorphism

$$
\begin{aligned}
& \mathcal{A} / \mathcal{J} \longrightarrow \prod_{x \in X} \mathcal{A}_{x}, \\
& \sigma(A) \longmapsto\left\{p_{x}(A)\right\}_{x \in X}
\end{aligned}
$$

of the symbol algebra.

(b) The homomorphism (2.1) is a monomorphism; that is, a family $A \in \mathcal{A}$ belongs to $\mathcal{J}$ (i.e., is compact and decays at infinity) if and only if all local representatives $p_{x}(A)$, $x \in X$, are zero.

(c) A family $A \in \mathcal{A}$ is elliptic (and hence Fredholm) with a parameter if and only if all local representatives $p_{x}(A), x \in X$, are invertible.

Proof. 1. First, let us prove that the algebra $\mathcal{A}$, the commutative subalgebra $\mathcal{C}=C(X)$, and the ideal $\mathcal{J}$ satisfy the assumptions of Proposition 3.1 in [15]. Specifically, we should verify that

(i) the restriction to $\mathcal{C}$ of an arbitrary irreducible representation of $\mathcal{A}$ is nonzero;

(ii) for two arbitrary distinct points $x_{1}, x_{2} \in X$, there exist elements $\varphi_{1}, \varphi_{2} \in C(X)$ such that $\varphi_{1}\left(x_{1}\right) \neq 0, \varphi_{2}\left(x_{2}\right) \neq 0$, and $\varphi_{1} \mathcal{A} \varphi_{2} \subset \mathcal{J}$

(iii) for an arbitrary irreducible representation $\pi$ of $\mathcal{J}$ and any $x \in X$, there exist elements $A \in \mathcal{J}$ and $\psi \in \mathcal{I}_{x}$ such that $\pi(A \psi) \neq 0$.

Condition (i) is trivial, since the subalgebra $\mathcal{C}$ contains the unit. Condition (ii) holds, since for $\varphi_{1,2}$ we can take functions supported in disjoint neighborhoods of $x_{1,2}$; then the desired inclusion follows from (1.1).

To prove (iii), note that each irreducible representation $\pi$ of $\mathcal{J}$ is equivalent to the representation $\pi(A)=A(y) \in \mathcal{B} H$ for some $y=y(\pi) \in Y$ [7, Corollary 10.4.4]. 
Now take an element $A \in \mathcal{J}$ such that $A(y) \neq 0$ and a vector $u \in H$ such that $A(y) u \neq 0$. Since $\lim _{\varphi \in \mathcal{F}_{x}}(1-\varphi) u=u$ (assumption $2^{\circ}$ ), it follows that $A(y)(1-\varphi) u \neq 0$ for some $\varphi \in \mathcal{F}_{x}$, and it suffices to set $\psi=1-\varphi \in \mathcal{I}_{x}$.

2. Thus the assumptions of Proposition 3.1 in [15] are satisfied. The argument in the proof of this proposition shows that

$$
\widehat{\mathcal{A} / \mathcal{J}}=\bigcup_{x \in X} \widehat{\mathcal{A}}_{x}
$$

where $\widehat{\mathcal{B}}$ is the spectrum of the $C^{*}$-algebra $\mathcal{B}$, i.e., the set of equivalence classes of its irreducible representations.

Relation (2.2) is understood as follows. Irreducible representations of the quotient algebras $\mathcal{A} / \mathcal{J}$ and $\mathcal{A}_{x}=\mathcal{A} / \mathcal{J}_{x}$ can be treated as irreducible representations of the algebra $\mathcal{A}$ itself. Then the right- and left-hand sides of the relation are sets of equivalence classes of irreducible representations of $\mathcal{A}$, and the assertion is that these two sets coincide.

It follows from (2.2) that

$$
\mathcal{J}=\bigcap_{x \in X} \mathcal{J}_{x}
$$

Indeed, by [3, Corollary, p. 34] for each element $A \notin \mathcal{J}$ there exists an irreducible representation $\pi \in \widehat{\mathcal{A} / \mathcal{J}}$ such that $\pi(A) \neq 0$. It follows from (2.2) that $\pi \in \widehat{\mathcal{A} / \mathcal{J}_{x}}$ for some $x$, so that $\operatorname{ker} \pi \supseteq \mathcal{J}_{x}$ and $A \notin \mathcal{J}_{x}$. Conversely, if $A \notin \mathcal{J}_{x}$ for some $x$, then, reversing the argument, we obtain $A \notin \mathcal{J}$.

In turn, (2.3) readily implies (a) and (b).

3. Finally, to obtain (c), on both sides of (2.2) we use the fact that an element of a $C^{*}$-algebra is invertible if and only if so are its images under all irreducible representations of the algebra (e.g., see [14, Proposition 5.3]).

\subsection{Properties of local representatives}

One can naturally ask how the local representatives of elements $A \in \mathcal{A}$ can be described more precisely.

First, we give an explicit description of the ideal $\mathcal{J}_{x}$ (and hence of the quotient algebra $\mathcal{A}_{x}$ ). It turns out that two elements of $\mathcal{A}$ give rise to the same element of $\mathcal{A}_{x}$ if and only if they are equivalent with respect to the localizing class $\mathcal{F}_{x}$ in the sense of formula (2.4) below (cf. the definition in $[9, \S 5.1]$ ).

Proposition 4. The ideal $\mathcal{J}_{x}$ consists of elements $A \in \mathcal{A}$ such that

$$
\lim _{\varphi \in \mathcal{F}_{x}}\|A \varphi\|=0
$$

Proof. If an element $A \in \mathcal{A}$ satisfies (2.4), then

$$
A=\lim _{\varphi \in \mathcal{F}_{x}} A(1-\varphi)
$$


and since $1-\varphi \in \mathcal{I}_{x}$, we see that $A \in \mathcal{J}_{x}$. To prove the converse, note that $\mathcal{J}_{x}$ is by definition the closure of the set of elements representable as finite sums

$$
C=\sum_{n} A_{n} \varphi_{n} B_{n}, \quad A_{n}, B_{n} \in \mathcal{A}, \quad \varphi_{n} \in \mathcal{I}_{x}
$$

Commuting $B_{n}$ with $\varphi_{n}$ and observing that each $\varphi_{n}$ can be approximated in norm by elements of the form $\varphi_{n}(1-\psi), \psi \in \mathcal{F}_{x}$, we see that $C$ can be approximated in norm by elements of the form

$$
A=\widetilde{C}(1-\psi)+K, \quad \widetilde{C}=\sum A_{n} B_{n} \varphi_{n} \in \mathcal{A}, \quad K \in \mathcal{J}
$$

Thus the elements (2.6) are dense in $\mathcal{J}_{x}$, and it suffices to prove (2.4) for these elements. In this case,

$$
\lim _{\varphi \in \mathcal{F}_{x}} A \varphi=\lim _{\varphi \in \mathcal{F}_{x}} \widetilde{C}(1-\psi) \varphi+\lim _{\varphi \in \mathcal{F}_{x}} K \varphi .
$$

The first term is eventually zero, and it remains to prove that the limit of the second term is zero. Since $K=K(y) \rightarrow 0$ as $y \rightarrow \infty$, it follows that for each $\varepsilon>0$ there exist finitely many compact operators $K_{0}=0, K_{1}, \ldots, K_{N}, N=N(\varepsilon)$, such that for each $y \in Y$ the relation

$$
\left\|K(y)-K_{j}\right\| \leq \varepsilon
$$

is valid for some $j=j(y) \in\{0,1, \ldots, N\}$. It follows from condition $2^{\circ}$ and the compactness of $K_{j}$ that

$$
\lim _{\varphi \in \mathcal{F}_{x}} K_{j} \varphi=0
$$

Combining this with (2.7), we see that

$$
\left\|\lim _{\varphi \in \mathcal{F}_{x}} K \varphi\right\|=\sup _{y}\left\|\lim _{\varphi \in \mathcal{F}_{x}} K(y) \varphi\right\| \leq \varepsilon,
$$

and we arrive at the desired assertion, since $\varepsilon$ is arbitrary.

Remark 5. In (2.4), $\|A \varphi\|$ can be replaced with $\|\varphi A\|$ or even with the norm of the element $\sigma(A \varphi)=\sigma(\varphi A)$ in $\mathcal{A} / \mathcal{J}$.

Note that Theorem 3 does not describe the range of the homomorphism (2.1). General facts about homomorphisms of $C^{*}$-algebras only imply that this is a $C^{*}$-subalgebra of $\prod_{x} \mathcal{A}_{x}$ isomorphic to $\mathcal{A} / \mathcal{J}$. The following questions are natural to ask. Let $\left\{a_{x}\right\}_{x \in X}$ be a family of elements of the local algebras $\mathcal{A}_{x}$. Under what conditions can this family be obtained by the localization of some element of $\mathcal{A}$ ? Knowing the family, how can one reconstruct this element modulo the ideal $\mathcal{J}$ ?

The family $\left\{a_{x}\right\}$ can be viewed as a section of the projection $\bigsqcup_{x} \mathcal{A}_{x} \longrightarrow X$, where $\bigsqcup_{x} \mathcal{A}_{x}$ is the disjoint union of the sets $\mathcal{A}_{x}$. The topology on $\bigsqcup_{x} \mathcal{A}_{x}$ with respect to which the families obtained by localization are exactly the continuous sections of the projection was studied in the framework of general localization principles in $C^{*}$-algebras in many papers (e.g., see $[6,18]$ etc.). In our case, one can readily describe such families without using the above-mentioned general results. 
We introduce the following notation. Let $Q \subset X$ be an arbitrary subset, and let $B \in C(Y, \mathcal{B} H)$. By $\|B\|_{Q}$ we denote the norm of the restriction of $B$ to the subset $H_{Q} \subset H$ of elements supported in the closure of $Q$ :

$$
\|B\|_{Q}=\sup _{y \in Y}\left\|\left.B(y)\right|_{H_{Q}}: H_{Q} \longrightarrow H\right\| .
$$

For each element $a_{x}$, we take some representative $A_{x} \in a_{x}$.

Definition 6. The family $\left\{a_{x}\right\}$ is said to be continuous if for each $\varepsilon>0$ every point $x \in X$ has a neighborhood $U(\varepsilon, x)$ such that the following condition is satisfied:

$$
\left\|A_{x}-A_{x^{\prime}}\right\|_{U(\varepsilon, x) \cap U\left(\varepsilon, x^{\prime}\right)} \leq \varepsilon \quad \text { for any } x, x^{\prime} \in X .
$$

It is easily seen that this condition is independent of the choice of representatives $A_{x} \in a_{x}$ (although the neighborhoods $U(\varepsilon, x)$ may depend on this choice).

Indeed, it follows from Proposition 4 that if $\widetilde{A}_{x}$ are some other representatives of $a_{x}$, then for each $x$ then norm $\left\|A_{x}-\widetilde{A}_{x}\right\|_{U}$ can be made as small as desired if we take a sufficiently small neighborhood $U$ of the point $x$ (where the corresponding element $\varphi \in \mathcal{F}_{x}$ is equal to unity).

Proposition 7. The family $\left\{a_{x}\right\}$ is the localization of some operator $A \in \mathcal{A}$ if and only if it is continuous.

Proof. Let $a_{x}=\sigma_{x}(A), x \in X$. Then one can take $A_{x}=A$ and $U(\varepsilon, x)=X$ for all $x \in X$ and $\varepsilon>0$. Then, obviously, $\left\|A_{x}-A_{x^{\prime}}\right\|=0$, so that condition (2.8) holds.

Conversely, if $\left\{a_{x}\right\}$ is a continuous family of local representatives, then an element $A \in \mathcal{A}$ such that

$$
a_{x}=p_{x}(A) \text { for all } x \in X,
$$

can be constructed by the following method. For each element $a_{x}$, take some representative $A_{x} \in a_{x}$. Next, for each $\varepsilon>0$ take a finite continuous nonnegative partition of unity $1=\sum_{x \in X} \varphi_{\varepsilon x}$ on $X$ subordinate to the cover $\{U(\varepsilon, x)\}$ in Definition [ 6 and set

$$
A^{(\varepsilon)}=\sum_{x} \varphi_{\varepsilon x} A_{x}
$$

We claim that there exists a limit (independent of the ambiguity in the construction)

$$
B=\lim _{\varepsilon \rightarrow 0} \sigma\left(A^{(\varepsilon)}\right) \in \mathcal{A} / \mathcal{J}
$$

and that (2.9) holds for arbitrary $A \in \mathcal{A}$ such that $\sigma(A)=B$. Indeed,

$$
A^{(\varepsilon)}-A^{(\delta)}=\sum_{x, y} \varphi_{\varepsilon x} \varphi_{\delta y}\left(A_{x}-A_{y}\right)
$$

Let us use the following lemma. 
Lemma 8. Let $f_{j} \in C(X), j=1, \ldots, N$, be nonnegative function, and let $A_{j} \in \mathcal{A}$ be some elements. Then

$$
\left\|\sigma\left(\sum_{j=1}^{N} f_{j} A_{j}\right)\right\|_{\mathcal{A} / \mathcal{J}} \leq\left[\max _{x \in X} \sum_{j=1}^{N} f_{j}(x)\right] \max _{j=1, \ldots, N}\left\|A_{j}\right\|_{\operatorname{supp} f_{j}} .
$$

The proof of this lemma is similar to that of Theorem 4.1 in [9]. It follows from the lemma that

$$
\left\|\sigma\left(A^{(\varepsilon)}\right)-\sigma\left(A^{(\delta)}\right)\right\|_{\mathcal{A} / \mathcal{J}} \leq \max \{2 \varepsilon, 2 \delta\}
$$

so that $\sigma\left(A^{(\varepsilon)}\right)$ is a Cauchy sequence in $\mathcal{A} / \mathcal{J}$ and hence has a limit, $B$. Using relations like (2.10), one can readily show that this limit is independent of the choice of partitions of unity.

Let $\sigma(A)=B$; let us show that (2.9) holds. We take a point $x_{0} \in X$ and choose partitions of unity such that $\varphi_{\varepsilon x_{0}}(x)=1$ in some neighborhood (depending on $\varepsilon$ ) of $x_{0}$. Then, obviously, $p_{x_{0}} A^{(\varepsilon)}=a_{x_{0}}$. Moreover,

$$
A=\lim _{\varepsilon \rightarrow 0}\left(A^{(\varepsilon)}+K^{(\varepsilon)}\right),
$$

where the $K^{(\varepsilon)}$ are appropriate elements of the ideal $\mathcal{J}$. Then

$$
p_{x_{0}}(A)=\lim _{\varepsilon \rightarrow 0} p_{x_{0}}\left(A^{(\varepsilon)}+K^{(\varepsilon)}\right)=\lim _{\varepsilon \rightarrow 0} p_{x_{0}}\left(A^{(\varepsilon)}\right)=a_{x_{0}} .
$$

The proof of the proposition is complete.

Summarizing, we obtain the following theorem.

Theorem 9. The set $\Sigma \subset \bigsqcup_{x \in X} \mathcal{A}_{x}$ of continuous families is a $C^{*}$-algebra with respect to the norm

$$
\left\|\left\{a_{x}\right\}\right\|=\sup _{x}\left\|a_{x}\right\|_{\mathcal{A}_{x}} .
$$

The mapping $\sigma(A) \longmapsto\left\{p_{x}(A)\right\}$ is a well-defined isometric isomorphism of the algebra $\mathcal{A} / \mathcal{J}$ onto the algebra $\Sigma$.

\section{Cones and infinitesimal operators}

An efficient application of Theorem 9 to general local operators is hindered by the fact that the local algebras $\mathcal{A}_{x}$ are not naturally realized as operator algebras. In particular, one cannot choose a "canonical" representative $A_{x}$ of $a_{x}$ so as to verify the invertibility of $a_{x}$ conveniently. However, if $X$ bears some additional structures, then in $\mathcal{A}$ there exist subalgebras for which the corresponding local algebras are naturally realized as operator algebras. In this section, we describe a relevant construction for the case in which the additional structure is the "tangent cone" to $X$. For the case of operators without parameter, a close construction can be found in [2, Chap. 4]. 


\subsection{Operators on the cone}

By a cone we mean a noncompact locally compact Hausdorff space $K$ with a distinguished point $0 \in K$ (the vertex) and with continuous one-parameter multiplicative dilation group

$$
g_{\lambda}: K \longrightarrow K, \quad \lambda \in \mathbb{R}_{+}
$$

which leaves the vertex fixed $\left(g_{\lambda}(0)=0\right)$ and has the following property: for each compact set $Q \subset K$ and each neighborhood of $V$,

$$
g_{\lambda}(Q) \subset V \text { for all sufficiently small } \lambda \in \mathbb{R}_{+} \text {. }
$$

it follows that the family of functions

$$
\varphi_{\lambda}(x)=\varphi\left(g_{\lambda} x\right), \quad x \in K, \quad \lambda \rightarrow \infty,
$$

is cofinal in $\mathcal{F}_{0}$ for each $\varphi \in \mathcal{F}_{0}$, where $\mathcal{F}_{0}$ is the set of compactly supported continuous functions $\varphi(x)$ on $K$ such that $0 \leq \varphi(x) \leq 1$ and $\varphi(x)=1$ in some neighborhood (depending on $\varphi$ ) of the vertex.

Next, let a Hilbert space $\widetilde{H}$ be a $*$-module over the $C^{*}$-algebra $C_{0}(K)$ of continuous functions on $K$ decaying at infinity, and suppose that the strong closure of the image of $C_{0}(K)$ in $\mathcal{B} \widetilde{H}$ contains the identity operator and assumption $2^{\circ}$ holds. Finally, let

$$
U_{\lambda}: \widetilde{H} \longrightarrow \widetilde{H}, \quad \lambda \in \mathbb{R}_{+}, \quad U_{\lambda} U_{\mu}=U_{\lambda \mu},
$$

be a strongly continuous one-parameter group of unitary operators in $\widetilde{H}$ related to the action of $C_{0}(K)$ by the formula

$$
U_{\lambda} \varphi(x) U_{\lambda}^{-1}=\varphi\left(g_{\lambda} x\right), \quad \lambda \in \mathbb{R}_{+}, \quad x \in K
$$

Proposition 10. The group $U_{\lambda}$ weakly converges to zero in $\widetilde{H}$ as $\lambda \rightarrow 0$ as well as as $\lambda \rightarrow \infty$.

Proof. It suffices to prove the weak convergence to zero as $\lambda \rightarrow \infty$; the second assertion then follows, since the group $U_{\lambda}$ is unitary. Next, by the Banach-Steinhaus theorem it suffices to prove the weak convergence on the dense subset of elements of the form $\varphi u$, where $u \in \widetilde{H}$ and $\varphi \in \mathcal{F}_{0}$. We have

$$
\left(v, U_{\lambda} \varphi u\right)=\left(v, U_{\lambda} \varphi U_{\lambda}^{-1} U_{\lambda} u\right)=\left(v, \varphi_{\lambda} U_{\lambda} u\right)=\left(\varphi_{\lambda} v, U_{\lambda} u\right) \rightarrow 0 \quad \text { as } \lambda \rightarrow \infty
$$

since $\varphi_{\lambda} v \stackrel{s}{\longrightarrow} 0$ by assumption $2^{\circ}\left(\varphi_{\lambda}\right.$ is cofinal in $\left.\mathcal{F}_{0}\right)$ and the family $U_{\lambda} u$ is uniformly bounded.

Suppose that $\mathbb{R}_{+}$also acts by homeomorphisms on the parameter space $Y$. This action will be denoted by $(\lambda, y) \longmapsto \lambda y$.

By $\mathcal{L} \subset C(Y, \mathcal{B} \widetilde{H})$ we denote the closed $C^{*}$-subalgebra of families $B$ such that

$$
[B, \varphi] \in \mathcal{J}=C_{0}(Y, \mathcal{K} \widetilde{H}) \quad \text { for each } \varphi \in C_{0}(K)
$$


Next, $\mathcal{J}_{0} \subset \mathcal{L}$ is the closed $*$-ideal in $\mathcal{L}$ consisting of the families $B \in \mathcal{L}$ such that $B \varphi \in \mathcal{J}$ for each $\varphi \in C_{0}(K) .{ }^{1}$ Elements of this ideal will be called almost compact operators with a parameter. An element $B \in \mathcal{L}$ is said to be homogeneous if

$$
U_{\lambda}^{-1} B(\lambda y) U_{\lambda}=B(y) \text { for all } \lambda \in \mathbb{R}_{+} .
$$

The set of all homogeneous elements will be denoted by $\mathcal{L}_{\infty}$. Obviously, this is a $C^{*}$ subalgebra in $\mathcal{L}$.

\section{Proposition 11.}

$$
\mathcal{L}_{\infty} \cap \mathcal{J}_{0}=\{0\}
$$

Proof. Let $B \in \mathcal{L}_{\infty} \cap \mathcal{J}_{0}$. Consider the family $B \varphi$, where $\varphi \in \mathcal{F}_{0}$. Obviously, if $\psi \in \mathcal{F}_{0}$, then for $\lambda \gg 1$ we have $\psi \varphi_{\lambda}=\varphi_{\lambda}$ and

$$
\|B \varphi\|=\left\|U_{\lambda} B \varphi U_{\lambda}^{-1}\right\|=\sup _{y}\left\|B(\lambda y) \psi \varphi_{\lambda}\right\| .
$$

The family $B(y) \psi$ is contained in the ideal $\mathcal{J}$ and hence can be approximated with arbitrary accuracy by a step function of $y$ assuming finitely many compact values. Since $\varphi_{\lambda} \stackrel{s}{\rightarrow} 0$, we conclude that the right-hand side of the last relation tends to zero as $\lambda \rightarrow \infty$, and hence $B \varphi=0$ for each $\varphi \in \mathcal{F}_{0}$. Taking a sequence of functions $\varphi$ strongly convergent to the identity operator, we obtain $B=0$, as desired.

\subsection{Infinitesimal operators}

Now let $x \in X$, and let a homeomorphism

$$
f: \bar{V} \longrightarrow f(\bar{V}) \subset K
$$

of the closure of some neighborhood $V$ of $x$ on the closure of a neighborhood of the vertex of $K$ be given. Next, suppose that an isomorphism

$$
\gamma: H_{f(\bar{V})} \longrightarrow \widetilde{H}_{\bar{V}}
$$

of $*$-modules over the ring homomorphism $f^{*}: C(f(\bar{V})) \longrightarrow C(\bar{V})$ is given. (In what follows, we identify $V$ and $f(V)$ as well as the subspaces related by $\gamma$. ) For brevity, we refer to $K$ as the tangent cone to $X$ at $x$. (Needless to say, we do not claim uniqueness.)

Definition 12. An operator $A \in \mathcal{A}$ with a parameter is said to be infinitesimally localizable at the point $x$ along the tangent cone $K$ if the class $p_{x}(A) \in \mathcal{A}_{x}$ contains a representative of the form $\psi B \varphi$, where $B \in \mathcal{L}_{\infty}$ and $\psi, \varphi \in \mathcal{F}_{x}$ are function supported in $V$. The operator $B$ is called the infinitesimal operator for $A$ at $x$ and is denoted by

$$
B=i_{x}(A)
$$

Theorem 13. Definition 12 is consistent. The mapping $A \longmapsto i_{x}(A)$ is a $C^{*}$-algebra homomorphism and factors through the quotient algebra $\mathcal{A}_{x}$.

\footnotetext{
${ }^{1}$ It obviously suffices to impose this condition for $\varphi \in \mathcal{F}_{0}$.
} 
Proof. 1. First of all, let us prove the consistency, i.e., the fact that the element $i_{x}(A) \in$ $\mathcal{L}_{\infty}$ is uniquely determined. Indeed, let $B$ and $\widetilde{B}$ be two such elements, so that

$$
\psi B \varphi, \widetilde{\psi} \widetilde{B} \widetilde{\varphi} \in p_{x}(A) .
$$

Set $\Delta=B-\widetilde{B} \in \mathcal{L}_{\infty}$. Taking a function $\chi \in \mathcal{F}_{x}$ such that all four elements of $\mathcal{F}_{x}$ in the last formula are equal to unity on the support of $\chi$, we obtain

$$
\chi \Delta \chi=\chi(B-\widetilde{B}) \chi=\chi(\psi B \varphi-\widetilde{\psi} \widetilde{B} \widetilde{\varphi}) \chi \in \mathcal{J}_{x} .
$$

By applying Proposition 4, we obtain

$$
\lim _{\rho \in \mathcal{F}_{x}}\|\chi \Delta \chi \rho\|=0 .
$$

Take $\rho=\phi_{\lambda}$, where $\phi \in \mathcal{F}_{0}$. For sufficiently large $\lambda$, we have $\chi \phi_{\lambda}=\phi_{\lambda}$ and

$$
\left\|\phi_{\lambda} \Delta \phi_{\lambda}\right\| \leq\left\|\chi \Delta \chi \phi_{\lambda}\right\| \longrightarrow 0 \text {. }
$$

On the other hand, by passing to the cone and by using the homogeneity of the element $\Delta$, we obtain

$$
\begin{aligned}
\left\|\phi_{\lambda} \Delta \phi_{\lambda}\right\| & =\left\|U_{\lambda}^{-1} \phi_{\lambda} \Delta \phi_{\lambda} U_{\lambda}\right\|=\left\|\phi U_{\lambda}^{-1} \Delta U_{\lambda} \phi\right\| \\
& =\sup _{y}\|\phi \Delta(y / \lambda) \phi\|=\sup _{y}\|\phi \Delta(y) \phi\|=\|\phi \Delta \phi\| .
\end{aligned}
$$

Hence $\phi \Delta \phi=0$ for each $\phi \in \mathcal{F}_{0}$, and consequently,

$$
\Delta=\mathrm{s}_{\lambda \rightarrow 0} \lim _{\lambda} \Delta \phi_{\lambda}=0 .
$$

Now it is obvious that if $A \in \mathcal{J}_{x}$, then $i_{x}(A)=0$. Indeed, $0 \in p_{x}(A)$ in this case.

2. Let us verify that the mapping $i_{x}$ is multiplicative. (Additivity is obvious.) Let $A$ and $C$ be infinitesimally localizable operators. Then

$$
\begin{aligned}
A C-\psi i_{x}(A) i_{x}(C) \varphi=A C-\psi i_{x}(A) \chi^{2} i_{x}(C) \varphi+K & \\
& =\left(A-\psi i_{x}(A) \chi\right) C+\psi i_{x}(A) \chi\left(C-\chi i_{x}(C) \varphi\right)+K
\end{aligned}
$$

for some $\chi \in \mathcal{F}_{x}$ and $K \in \mathcal{J}$, and all terms on the right-hand side belong to $\mathcal{J}_{x}$. Thus the product $A C$ is infinitesimally localizable, and

$$
i_{x}(A C)=i_{x}(A) i_{x}(C) .
$$

3. Let us prove that the set of operators infinitesimally localizable at $x$ is closed. Using the same trick as in (3.3), we can show that for a homogeneous element $i_{x}(A) \in \mathcal{L}_{\infty}$ one always has

$$
\left\|i_{x}(A)\right\|=\left\|\varphi i_{x}(A) \varphi\right\|, \quad \varphi \in \mathcal{F}_{0}
$$

On the other hand,

$$
\left\|\varphi\left(A-i_{x}(A)\right) \varphi\right\| \stackrel{\varphi \in \mathcal{F}_{x}}{\longrightarrow} 0 .
$$

It follows that

$$
\left\|i_{x}(A)\right\| \leq\|A\| .
$$

Since the set of homogeneous elements is a $C^{*}$-algebra and, in particular, is closed, we see that so is the set of infinitesimally localizable operators. The proof is complete. 
Let $Z$ be a set of pairs of the form $(x, K)$, where $x \in X$ and $K$ is a tangent cone at $x$. We define a projection $\pi: Z \longrightarrow X$ by setting $\pi(x, K)=x$. (We do not exclude the case in which several tangent cones are given at some point $x$.) We assume that $\pi(Z)=X$. We denote by $\mathcal{A}_{Z}$ the subset of $\mathcal{A}$ formed by the elements infinitesimally localizable at $x$ along $K$ for each pair $(x, K) \in Z$. The preceding argument readily implies the following theorem.

Theorem 14. The set $\mathcal{A}_{Z}$ is a $C^{*}$-algebra. The mapping

$$
A \longmapsto\left\{i_{x}(A)\right\}_{(x, K) \in Z}
$$

factors through $\mathcal{A}_{Z} / \mathcal{J}$ and is a monomorphism.

\subsection{The decomposition problem for infinitesimal operators}

If a local operator $A \in \mathcal{A}$ with a parameter has an infinitesimal operator $i_{x}(A)$ (along a given cone) at a point $x$ and if this infinitesimal operator is invariant with respect to some transformation group, then, passing to the Fourier transform associated with this group, one can decompose the operator $i_{x}(A)$ into a direct sum (or direct integral) over irreducible representations of the group. This was indicated, say, in [2, Chap. 4]. A typical example is given by classical zero-order $\Psi \mathrm{DO} A$ on a smooth manifold $X$. Here the tangent cone is just the tangent space $T_{x} X$ at $x$ (a neighborhood of zero in the tangent space is mapped onto a neighborhood of the point in the manifold, say, with the help of local coordinates with origin at $x$ ), the infinitesimal operator $i_{x}(A)$ is a translation invariant operator in $L^{2}\left(T_{x} X\right)$ and hence has the form

$$
i_{x}(A)=f\left(x,-i \frac{\partial}{\partial t}\right)
$$

(where $x$ is a parameter and the variables $t$ are coordinates on $T_{x} X$ ), and the Fourier transform associated with the translation group is none other than the ordinary Fourier transform and takes the infinitesimal operator to the operator of multiplication by a function $f(x, \xi), \xi \in T_{x}^{*} X$. This function is called the (principal) symbol of $A$, is denoted by $\sigma(A)(x, \xi)$, is continuous and zero-order homogeneous with respect to $\xi$ for $\xi \neq 0$, and has a jump discontinuity at the zero section of the cotangent bundle $T^{*} X$.

What are the conditions under which the infinitesimal operator proves to be invariant under some transformation group, so that the Fourier transform gives a "symbol"? We give an answer in the next subsection.

\subsection{Symbols in the small}

Let $K$ be a cone (whose points will be denoted by $z$ and the action of elements $\lambda \in \mathbb{R}_{+}$ by $\lambda z$ ), and let $\widetilde{H}$ be a Hilbert space that is a $*$-module over $C_{0}(K)$ and is equipped with a unitary group associated with dilations. (Here the group will be denoted by $\varkappa_{\lambda}$.) Next, suppose that an open subset $U \subset X$ is homeomorphically mapped onto a neighborhood of the point $(0,0)$ in the Cartesian product $\mathbb{R}^{k} \times K$. (For elements of $U$, 
we use the coordinates $(t, z), t \in \mathbb{R}^{k}, z \in K$.) Finally, let the subspace $H_{U}$ be identified via an isomorphism respecting the module structure with the corresponding subspace of the Hilbert space $\mathfrak{H}=L^{2}\left(\mathbb{R}^{k}, \widetilde{H}\right.$ ) (on which the algebra $C_{0}\left(\mathbb{R}^{k} \times K\right.$ ) acts naturally, i.e., pointwise with respect to the argument $t \in \mathbb{R}^{k}$ ). Then for each point of the form $(x, 0) \in U$ the product $\mathbb{R}^{k} \times K$ can be viewed as the tangent cone with vertex $(x, 0)$, the action of $\mathbb{R}_{+}$given by the formula

$$
g_{x, \lambda}(t, z)=(x+\lambda(t-x), \lambda z), \quad \lambda \in \mathbb{R}_{+},
$$

and the one-parameter unitary group in $\mathfrak{H}$ given by the formula ${ }^{2}$

$$
U_{x, \lambda} f(t)=\lambda^{k / 2}\left(\varkappa_{\lambda} f\right)(x+\lambda(t-x)) .
$$

A straightforward computation shows that the group interacts with the action of $C_{0}\left(\mathbb{R}^{k} \times\right.$ $K$ ) by the desired formula

$$
U_{x, \lambda} \varphi(t, z) U_{x, \lambda}^{-1}=\varphi\left(g_{x, \lambda}(t, z)\right) .
$$

We are interested in infinitesimal operators of a given operator $A$ with a parameter at the points $(x, 0) \in U$. For brevity, we denote them by $a_{x}=i_{x}(A)$ instead of $i_{(x, 0)}(A)$. They all act in the space $\mathfrak{H}$, but the cone is different for each $x$. (The cone vertex and the dilation groups depend on $x$ ). These infinitesimal operators are determined by the homogeneity condition (3.2) and the convergence

$$
\lim _{\phi \in \mathcal{F}_{x}}\left\|\psi\left(A-a_{x}\right) \phi\right\|=0
$$

where $\psi \in C_{0}(U)$ is an arbitrary cutoff function. We consider only $x$ close to zero; hence it can be assumed that $A$ has already been multiplied on the left and on the right by cutoff functions and is well defined in $\mathfrak{H}$, so that the cutoff factor $\psi$ in this condition can be omitted. Next, we represent the function $\phi \in \mathcal{F}_{x}$ in the convergence condition in the form

$$
\phi=T_{-x} \varphi T_{x},
$$

where $\varphi \in \mathcal{F}_{0}$ and $T_{x}$ is the translation operator

$$
T_{x} f(t, z)=f(t+x, z) .
$$

Then the condition acquires the form

$$
\lim _{\varphi \in \mathcal{F}_{0}}\left\|\left(A-a_{x}\right) T_{-x} \varphi T_{x}\right\|=0
$$

which will be used in what follows. ${ }^{3}$

Theorem 15. (i) If $A \in \mathcal{A}$ is an operator with a parameter such that the infinitesimal operators $a_{x}=i_{x}(A)$ exist at all points $(x, 0) \in U$ and the convergence in (3.5) is locally

\footnotetext{
${ }^{2}$ Here the factor $\lambda^{k / 2}$ ensures that the group is unitary.

${ }^{3}$ The factor $T_{-x} \varphi T_{x}$ can also be placed on the left; the results will be the same.
} 
uniform with respect to $x$, then the infinitesimal operators $a_{x}$ commute with translations with respect to $x$, i.e., satisfy

$$
\left[a_{x}, T_{\tau}\right]=0 \text { for any } \tau \text { and } x,
$$

and depend on $x$ continuously in the norm $\|\cdot\|$. The set of operators satisfying these conditions is a $C^{*}$-subalgebra in $\mathcal{A}$ and contains the ideal $\mathcal{J}$.

(ii) Conversely, suppose that the infinitesimal operators $a_{x}$ for an operator $A \in \mathcal{A}$ exist, commute with translations, and continuously depend on the parameter $x$. Then the convergence in (3.5) is locally uniform with respect to $x$.

Proof. First, let us prove (i).

1. Let us verify that the infinitesimal operators are translation invariant. It suffices to do this for $x=0$. In (3.5), we replace the arbitrary function $\varphi$ by $\varphi_{\lambda}=U_{\lambda} \varphi U_{\lambda}^{-1}$ (see (3.1)), where $U_{\lambda} \equiv U_{0, \lambda}$ and the function $\varphi \in \mathcal{F}_{0}$ is arbitrary but fixed. Then we speak of convergence locally uniform with respect to $x$ as $\lambda \rightarrow \infty$. First, we write out some relations between our groups, which can be verified by a straightforward computation:

$$
U_{x, \lambda}=T_{-x} U_{0, \lambda} T_{x}, \quad U_{\lambda} T_{x}=T_{x / \lambda} U_{\lambda}=T_{x} U_{x, \lambda} .
$$

Throughout the following, we assume that $x$ varies in a neighborhood of zero; moreover, $\lambda>1$, so that $x / \lambda$ lies in the same neighborhood. Since condition (3.5) is uniform with respect to $x$, we can replace $x$ by $x / \lambda$ in it. Separately substituting also $x=0$ into this condition, we obtain (the arrow means convergence in the norm $\|\cdot\|$ as $\lambda \rightarrow \infty$ )

$$
\left(A-a_{0}\right) \varphi_{\lambda} \rightarrow 0, \quad\left(A-a_{x / \lambda}\right) T_{-x / \lambda} \varphi_{\lambda} T_{x / \lambda} \rightarrow 0 .
$$

We multiply the equations in (3.7) on the left and on the right by the unitary operators $T_{ \pm x / \lambda}$ and obtain

$$
T_{x / \lambda}\left(A-a_{0}\right) T_{-x / \lambda} T_{x / \lambda} \varphi_{\lambda} T_{-x / \lambda} \rightarrow 0, \quad T_{x / \lambda}\left(A-a_{x / \lambda}\right) T_{-x / \lambda} \varphi_{\lambda} \rightarrow 0 .
$$

Take a function $\chi \in \mathcal{F}_{0}$ such that

$$
\chi \varphi=\chi T_{x} \varphi T_{-x}=\chi
$$

for all $x$ in our neighborhood. This can be done if the space is sufficiently small. The function $\chi$ can be assumed to be an arbitrary given element of $\mathcal{F}_{0}$. (Given $\chi$, there always exists an appropriate $\varphi$.) Multiplying both equations in (3.8) on the right by $\chi_{\lambda}$ and using (3.6), we obtain

$$
T_{x / \lambda}\left(A-a_{0}\right) T_{-x / \lambda} \chi_{\lambda} \rightarrow 0, \quad T_{x / \lambda}\left(A-a_{x / \lambda}\right) T_{-x / \lambda} \chi_{\lambda} \rightarrow 0,
$$

and then, subtracting one from the other,

$$
T_{x / \lambda}\left(a_{x / \lambda}-a_{0}\right) T_{-x / \lambda} \chi_{\lambda} \rightarrow 0 .
$$

In (3.9), we use the homogeneity property

$$
a_{0}(y)=U_{\lambda} a_{0}(y / \lambda) U_{\lambda}^{-1}, \quad a_{x / \lambda}(y)=U_{x / \lambda, \lambda} a_{x / \lambda}(y / \lambda) U_{x / \lambda, \lambda}^{-1}
$$


of infinitesimal operators. By substituting this into the left-hand side of (3.9) and by using relations (3.6), we obtain

$$
\begin{aligned}
T_{x / \lambda}\left(a_{x / \lambda}(y)-a_{0}(y)\right) & T_{-x / \lambda} \chi_{\lambda} \\
& =\left[U_{\lambda} T_{x / \lambda} a_{x / \lambda}(y / \lambda) T_{-x / \lambda} U_{\lambda}^{-1}-T_{x / \lambda} U_{\lambda} a_{0}(y / \lambda) U_{\lambda}^{-1} T_{-x / \lambda}\right] \chi_{\lambda} \\
& =U_{\lambda}\left[T_{x / \lambda} a_{x / \lambda}(y / \lambda) T_{-x / \lambda}-T_{x} a_{0}(y / \lambda) T_{-x}\right] \chi U_{\lambda}^{-1} \rightarrow 0 .
\end{aligned}
$$

The left- and rightmost unitary factors $U_{\lambda}^{ \pm 1}$ can be omitted. Next, since the norm $\|\cdot\|$ involves the supremum over $y$, we can replace the argument $y / \lambda$ of infinitesimal families by $y$, thus obtaining

$$
\left[T_{x / \lambda} a_{x / \lambda}(y) T_{-x / \lambda}-T_{x} a_{0}(y) T_{-x}\right] \chi \rightarrow 0 .
$$

It follows from (3.10) that the operator $T_{x / \lambda} a_{x / \lambda}(y) T_{-x / \lambda} \chi$ converges in the norm to the operator $T_{x} a_{0}(y) T_{-x} \chi$ as $\lambda \rightarrow \infty$. On the other hand, one can readily show that under the conditions of the theorem it strongly converges to the operator $T_{0} a_{0}(y) T_{0} \chi=a_{0}(y) \chi$.

Indeed, let us show that the operator function

$$
\widetilde{a}_{x}=T_{x} a_{x} T_{-x} \chi
$$

is strongly continuous. It follows from the definitions that

$$
\lim _{\lambda \rightarrow \infty} F(x, \lambda)=\widetilde{a}_{x}
$$

locally uniformly with respect to $x$ in the norm and hence in the strong sense, where the operator function

$$
F(x, \lambda)=U_{\lambda}^{-1} T_{x} A T_{-x} U_{\lambda} \chi
$$

is strongly continuous in $x$. Thus the desired assertion follows from the continuity of a uniform limit of continuous functions.

The limits should coincide, and hence

$$
T_{x} a_{0}(y) T_{-x} \chi=a_{0}(y) \chi
$$

for each $\chi \in \mathcal{F}_{0}$. Since vectors of the form $\chi u$ are dense in $\mathfrak{H}$ (recall that the identity operator lies in the strong closure of the algebra $C_{0}\left(\mathbb{R}^{k} \times K\right)$ ), we obtain the desired assertion

$$
T_{x} a_{0} T_{-x}=a_{0}
$$

2. Let us prove that the operator $a_{x}$ continuously depends on $x$ for $x=0$. For a given $\varepsilon>0$, for each point $(x, 0) \in U$ we take a neighborhood $U(\varepsilon, x)$ in accordance with Proposition 7 . We consider only points $x$ that are so close to zero that $(x, 0) \in$ $U(\varepsilon, 0)$; then the intersection $O=U(\varepsilon, 0) \cap U(\varepsilon, x)$ is necessarily a nonempty open set (a neighborhood of the point $(x, 0))$. By Proposition [7] $\left\|\psi\left(a_{x}-a_{0}\right)\right\|_{O}<\varepsilon$, where $\psi \in \mathcal{F}_{0}$ is the function equal to unity in $O$. Since the operator $a_{x}-a_{0}$ is translation invariant, we obtain

$$
\left\|\widetilde{\psi}\left(a_{x}-a_{0}\right)\right\|_{\widetilde{O}}<\varepsilon
$$


where $\widetilde{O}=T_{-x}(O)$ is a neighborhood of $(0,0)$ and the translate $\widetilde{\psi}$ of $\psi$ by $-x$ again lies in $\mathcal{F}_{0}$. Multiplying this on the right by a function $\varphi \in \mathcal{F}_{0}$ supported in $\widetilde{O}$, we obtain

$$
\left\|\widetilde{\psi}\left(a_{x}-a_{0}\right) \varphi\right\|_{\widetilde{O}}<\varepsilon .
$$

The operator $B=a_{x}-a_{0}$ has the homogeneity property (3.2) with respect to the group $U_{\lambda}$. (For $a_{0}$ this is true by definition, and for $a_{x}$ this follows from the definition combined with the translation invariance.) Using the homogeneity, we make the supports of $\widetilde{\psi}$ and $\varphi$ arbitrarily large without changing the operator $B$. It remains to use the fact that the identity operator belongs to the strong closure of $C_{0}\left(\mathbb{R}^{k} \times K\right)$ to conclude that $\left\|a_{x}-a_{0}\right\|<\varepsilon$ for $x$ close to zero.

3. Now let us show that the elements of $\mathcal{J}$ satisfy the conditions of the theorem. For an element $A \in \mathcal{J}$, condition (3.5) can be rewritten as

$$
\lim _{\varphi \in \mathcal{F}_{0}}\left\|A T_{-x} \varphi\right\|=0 .
$$

(We have omitted the unitary factor $T_{x}$ that does not affect the norm and used the fact that $a_{x}=0$ for a compact operator.) Since $A$ is compact and the operator $T_{x}$, which is the adjoint of $T_{-x}$, is a strongly continuous function of $x$, it follows that the family $A_{x}=A T_{-x}$ is continuous in the norm and condition, and so the convergence in (3.13) is locally uniform in $x$.

4. Let us show that the set of elements satisfying the assumptions of item (i) is a *algebra. Assertion (ii) of the theorem, proved below, permits us to replace the assumptions of item (i) by those of item (ii), which obviously are respected by algebraic operations and the passage to the adjoint operator.

It remains to show that this set is closed. Let a sequence $A_{n} \in \mathcal{A}$ converge in $A$, and suppose that for each $n$ the convergence

$$
\lim _{\varphi \in \mathcal{F}_{0}}\left\|\left(A_{n}-a_{n x}\right) T_{-x} \varphi T_{x}\right\|=0
$$

is locally uniform in $x$. As was shown earlier (Theorem 13), the limit element $a$ is also infinitesimally localizable at each point $(x, 0)$, and moreover, $a_{x}=\lim _{n \rightarrow \infty} a_{n x}$. The convergence of $a_{n x}$ to $a_{x}$ is uniform, since (see (3.4))

$$
\left\|a_{n}-a_{n x}\right\| \leq\left\|A-A_{n}\right\| .
$$

Now it is obvious that the convergence in (3.5) for the limit operator $A$ is uniform.

Now let us prove (ii). We give only a brief scheme of the proof; the reader can readily reconstruct the details. We use the method in Proposition 7 to reconstruct the operator $A$ from its local representatives. Moreover, we choose the neighborhoods $U(\varepsilon,(\xi, z))$ of points $(\xi, z)$ with $z \neq 0$ (i.e., $z$ is not the cone vertex) so that they do not contain any points of the form $(x, 0)$. For the local representatives we take the elements $a_{x}$ for the points $(x, 0)$ and $A$ for all other points. Having constructed the approximations by this method, we have

$$
A=\lim _{\varepsilon \rightarrow 0}\left(A^{(\varepsilon)}+K^{(\varepsilon)}\right)
$$


where the $K^{(\varepsilon)}$ belong to $\mathcal{J}$. Using the assumptions imposed on the local representatives, we can readily verify that the operators $A^{(\varepsilon)}$ (and hence $A^{(\varepsilon)}+K^{(\varepsilon)}$ ) satisfy all assumptions of item (i) of the theorem; namely, they are infinitesimally localizable at each point $(x, 0)$, and the corresponding convergence (3.5) is locally uniform. But then, as was shown above, the uniform convergence holds also for the limit operator $A$.

The proof of the theorem is complete.

Thus the theorem gives the desired group commuting with infinitesimal operators. Now we can use the Fourier transform and decompose the infinitesimal operators into families of simpler operators depending on the parameter $\xi$. The following assertion establishes the corresponding result.

Proposition 16. Let $H$ be a (separable) Hilbert space, and let

$$
\widehat{B}: L^{2}\left(\mathbb{R}^{k}, H\right) \longrightarrow L^{2}\left(\mathbb{R}^{k}, H\right)
$$

be a bounded linear operator commuting with translations in $\mathbb{R}^{k}$ :

$$
\widehat{B} T_{x}=T_{x} \widehat{B}, \quad x \in \mathbb{R}^{k}, \quad \text { where } \quad\left[T_{x} u\right](t)=u(x+t) .
$$

Then $B$ can be represented in the form

$$
\widehat{B}=\overline{\mathcal{F}}_{\xi \rightarrow t} B(\xi) \mathcal{F}_{t \rightarrow \xi},
$$

where $\mathcal{F}$ is the Fourier transform and $B(\xi)$ is a strongly measurable ${ }^{4}$ operator-valued function ranging in $\mathcal{B H}$ and satisfying the estimate

$$
\underset{\xi}{\operatorname{ess} \sup }\|B(\xi)\|=\|\widehat{B}\|
$$

If the operator $\widehat{B}$ continuously depends on some parameters $\tau$, then $B(\xi)$ continuously depends on the same parameters:

$$
\underset{\xi}{\operatorname{ess} \sup }\left\|B_{\tau}(\xi)-B_{\tau_{0}}(\xi)\right\| \rightarrow 0 \quad \text { as } \tau \rightarrow \tau_{0}
$$

The proof will be given in the Appendix.

Remark 17. If a function $u \in L^{2}\left(\mathbb{R}^{k}, H\right)$ is such that its Fourier transform $\widetilde{u}$ lies in $L^{2}\left(\mathbb{R}^{k}, H\right) \cap L^{1}\left(\mathbb{R}^{k}, H\right)$, then $B \widetilde{u}$ lies in the same space, and hence the inverse Fourier transform is given by a Bochner integral and we can write

$$
[B u](t)=\left(\frac{1}{2 \pi}\right)^{n / 2} \int e^{i t \xi} B(\xi) \widetilde{u}(\xi) d \xi
$$

\footnotetext{
${ }^{4}$ In the separable case, strong and weak measurability are equivalent by the Pettis theorem [20].
} 
By the proposition we have just proved, to the infinitesimal operator $i_{x}(A)$ there corresponds an operator-valued function $\sigma(x, \xi, y)$ such that

$$
i_{x}(A)(y)=\overline{\mathcal{F}}_{\xi \rightarrow t} \sigma(x, \xi, y) \mathcal{F}_{t \rightarrow \xi},
$$

(here $(x, y)$ play the role of additional parameters $\tau$ ). Using the definition of the group $U_{\lambda}$ and the homogeneity property $i_{x}(A)(\lambda y)=U_{\lambda} i_{x}(A)(y) U_{\lambda}^{-1}$ and passing to the Fourier transform, we see that this operator-valued function satisfies the twisted homogeneity condition

$$
\sigma(x, \lambda \xi, \lambda y)=\varkappa_{\lambda} \sigma(x, \xi, y) \varkappa_{\lambda}^{-1}, \quad \lambda \in \mathbb{R}_{+} .
$$

Definition 18. The function $\sigma(x, \xi, y)$ is called the symbol of the operator $A$ with parameter $y \in Y$ on the domain of $\mathbb{R}^{k}$ embedded in $X$ and will be denoted by $\sigma(A)(x, \xi, y)$.

\section{Stratified manifolds}

In this section, we describe the class of manifolds on which elliptic theory will be studied (cf., e.g., $[5,13,15])$.

\subsection{Definition of stratified manifolds}

Let $\mathcal{M}$ be a Hausdorff locally compact topological space with a filtration of length $k$, i.e., a decreasing finite chain of closed subspaces of the form

$$
\mathcal{M} \equiv \mathcal{M}_{0} \supset \mathcal{M}_{1} \supset \cdots \supset \mathcal{M}_{k}
$$

The subspaces $\mathcal{M}_{j}$ will be called closed strata, the sets

$$
\mathcal{M}_{j}^{\text {o def }} \mathcal{M}_{j} \backslash \mathcal{M}_{j+1}, \quad j=0, \ldots, k
$$

will be called open strata, ${ }^{5}$ and we shall always assume that the open strata are smooth manifolds without boundary.

The space $\mathcal{M}$ will be called a stratified manifold of length $k$ if the following additional structure is specified on it. The definition of this structure involves induction over $k$; to define a stratified manifold of length $k$, one should have the definition not only of a stratified manifold of length $l \leq k-1$ but also of a diffeomorphism of such manifolds, smoothly depending on parameters, and of the structure of a stratified manifold of length $l$ on the product of a stratified manifold of length $l$ by an open subset in $\mathbb{R}^{m}$. We start from $k=0$ (the basis of induction).

Definition 19. A stratified manifold $\mathcal{M}$ of length zero is a smooth manifold without boundary. Diffeomorphisms of smooth manifolds (possibly, smoothly depending on parameters) and the structure of a smooth manifold on the product of $\mathcal{M}$ by an open subset in $\mathbb{R}^{m}$ are defined in a standard way. A coordinate system of type 0 on $\mathcal{M}$ is a smooth coordinate system.

\footnotetext{
${ }^{5}$ For brevity, we adopt the convention that $\mathcal{M}_{k+1}=\varnothing$.
} 
Now we proceed to the general case of a space $\mathcal{M}$ with a filtration (4.1). By analogy with smooth manifolds, we define a stratified manifold with the use of an atlas, i.e., a set of coordinate systems related by admissible transition maps. On the smooth part $\mathcal{M}_{0}^{\circ}$ of $\mathcal{M}$, coordinate systems are the same as in the smooth case. However, in neighborhoods of points of the singularity set $\mathcal{M}_{1}$ the local models of the manifold are more complicated objects than open subsets of $\mathbb{R}^{m}$. Let $\Omega \equiv \Omega_{0} \supset \cdots \supset \Omega_{l}$ be a stratified manifold of length $l<k$. By $K_{\Omega}$ we denote the infinite cone

$$
K_{\Omega}=\left\{\overline{\mathbb{R}}_{+} \times \Omega\right\} /\{\{0\} \times \Omega\}
$$

with base $\Omega$. We denote its vertex by $\mathbf{0}$ and set

$$
K_{\Omega}^{\circ}=K_{\Omega} \backslash\{\mathbf{0}\} \equiv \mathbb{R}_{+} \times \Omega .
$$

Local models in a neighborhood of points of $\mathcal{M}_{1}$ are open subsets of the products $\mathbb{R}^{m} \times K_{\Omega}$. The "coordinates" in such a product will be denoted by $(x, r, \omega)$, where $x \in \mathbb{R}^{m}, r \in \mathbb{R}_{+}$, and $\omega \in \Omega$.

Let us proceed to precise statements.

A coordinate system of type $l$ on $\mathcal{M}$ (centered at a point $z \in \mathcal{M}_{l}^{\circ}$ ) is a smooth coordinate system on $\mathcal{M}_{0}^{\circ}$ in a neighborhood of $z$ if $l=0$, while for $0<l \leq k$ it is a homeomorphism $\varphi: U \longrightarrow V$, where

1) $U \subset \mathcal{M}$ is a neighborhood of $z$ that does not meet $\mathcal{M}_{s}$ for $l<s \leq k$;

2) $V$ is neighborhood of the point $(0, \mathbf{0})=\varphi(z)$ in the product $\mathbb{R}^{m} \times K_{\Omega}$, where $\Omega=\Omega^{(l)}$ is a compact stratified manifold of length $l-1$;

3) $\varphi\left(U \cap \mathcal{M}_{l}^{\circ}\right)=V \cap\left(\mathbb{R}^{m} \times\{\mathbf{0}\}\right)$, and the restriction $\left.\varphi\right|_{U \cap \mathcal{M}_{l}^{\circ}}$, treated as a mapping into $\mathbb{R}^{m}$, is a smooth coordinate system on $\mathcal{M}_{l}^{\circ}$.

4) for $s<l$, one has $\varphi\left(U \cap \mathcal{M}_{s}^{\circ}\right)=V \cap \widetilde{\Omega}_{s}$, where $\widetilde{\Omega}_{s}=\mathbb{R}^{m} \times \mathbb{R}_{+} \times \Omega_{s}$ are the strata of the stratified manifold $\mathbb{R}^{m} \times K_{\Omega}^{\circ}$ of length $l-1$.

Now let $\varphi_{i}: U_{i} \longrightarrow V_{i}, i=1,2$, be two coordinate systems of types $l_{1}$ and $l_{2}$ on $M$ such that $U_{1} \cap U_{2} \neq \varnothing$. Then we have the transition map $\varphi_{12}=\varphi_{1} \circ \varphi_{2}^{-1}$. There are two possible cases: (1) at least one of the numbers $l_{i}$ is less than $k$; (2) $l_{1}=l_{2}=k$. In the first case, both the domain and the range of $\varphi_{12}$ are stratified manifolds of length $l=\min \left\{l_{1}, l_{2}\right\}<k$ and, using the induction assumption, we say that the transition mapping $\varphi_{12}$ is admissible if it is a diffeomorphism of stratified manifolds of length $l$. In the second case, we have $\varphi_{12}: W \longrightarrow \widetilde{W}$, where $W, \widetilde{W} \subset \mathbb{R}^{m} \times K_{\Omega}$ are some open domains and $\varphi_{12}\left(W \cap\left(\mathbb{R}^{m} \times K_{\Omega}^{\circ}\right)\right)=\widetilde{W} \cap\left(\mathbb{R}^{m} \times K_{\Omega}^{\circ}\right)$. We say that $\varphi_{12}$ is admissible if the restriction $\left.\varphi_{12}\right|_{W \cap\left(\mathbb{R}^{m} \times K_{\Omega}^{\circ}\right)}$ is a diffeomorphism of stratified manifolds of length $k-1$ and in a sufficiently small neighborhood of the set $\mathbb{R}^{m} \times\{\mathbf{0}\}$ the mapping $\varphi_{12}$ has the form

$$
\varphi_{12}(x, r, \omega)=(\widetilde{\varphi}(x), r, \psi(x, \omega)),
$$

where $\widetilde{\varphi}$ is a diffeomorphism and $\psi(x, \cdot): \Omega \longrightarrow \Omega$ is a diffeomorphism of a stratified manifolds $\Omega$ of length $k-1$ and smoothly depends on the parameters $x$.

It is easily seen that the composition of admissible transition maps is again admissible. (The mapping with empty domain is admissible by definition.) 
Definition 20. The structure of a stratified manifold of length $k$ on $\mathcal{M}$ is a maximal set of coordinate systems $(U, \varphi)$ of types $0, \ldots, k$ such that the union of all these $U$ covers $\mathcal{M}$ and all transition maps between coordinate systems are admissible.

If $\mathcal{M}$ is a stratified manifold of length $k$ and $W$ is a domain in $\mathbb{R}^{m}$, then the structure of a stratified manifold $k$ on $W \times \mathcal{M}$ is defined as follows: one considers all possible coordinate systems of the form id $\times \varphi: W \times U \longrightarrow W \times V$, where $\varphi: U \longrightarrow V$ is a coordinate system on $\mathcal{M}$, and the resulting atlas is embedded in a (uniquely determined) maximal atlas.

If $\mathcal{M}$ and $\mathcal{N}$ are stratified manifold of length $k$, then a diffeomorphism of $\mathcal{M}$ onto $\mathcal{N}$ is a homeomorphism $f: \mathcal{M} \longrightarrow \mathcal{N}$ preserving the structure of a stratified manifold (and hence having the form of admissible transition maps in coordinate systems).

We say that a diffeomorphism $f: \mathcal{M} \longrightarrow \mathcal{N}$ smoothly depends on parameters if the admissible transition maps representing it in coordinate systems smoothly depend on these parameters.

Remark 21. Note that admissible transition maps include only diffeomorphisms of stratified manifolds of length $\leq k-1$, for which the notion of smooth dependence on parameters is known by the induction assumption.

Remark 22 . One can readily see that each closed stratum $\mathcal{M}_{j}$ of a stratified manifold of length $k$ is itself a stratified manifold (of length $k-j$ ).

\subsection{Generating vector fields of families of diffeomorphisms}

If $f_{\tau}: \mathcal{M} \longrightarrow \mathcal{M}, \tau \in[0,1], f_{0}=$ id, is a smooth family of diffeomorphisms of a stratified manifold $\mathcal{M}$, then on the dense open set $\mathcal{M}^{\circ}=\mathcal{M} \backslash \mathcal{M}_{1}$ this family defines a vector field $V(z), z \in \mathcal{M}^{\circ}$, (depending on the parameter $\tau$ ), from which the family can be reconstructed as the solution of the ordinary differential equation $d z / d \tau=V(z)$. By our definition of diffeomorphisms, such a vector field has the following properties:

1) in a coordinate system of type 0 , this is an arbitrary smooth vector field;

2 ) in a coordinate system of type $l>0$, for sufficiently small $r$ the field has the form $V(x, r, \omega)=\left(V_{1}(x), 0, V_{2}(x, \omega)\right)$, where $\left(V_{1}(x), V_{2}(x, \omega)\right)$ is a field with similar properties 1), 2) on the stratified manifold $\mathbb{R}^{m} \times \Omega$ of length $l-1$.

Conversely, each field with these properties generates (at least locally, and globally on a compact stratified manifold) a family of diffeomorphisms. The space of vector fields defined by induction on $k$ via properties 1) and 2) on a stratified manifold $\mathcal{M}$ of length $k$ will be denoted by $\mathcal{V}(\mathcal{M})$.

\subsection{The blow-up}

For each stratified manifold, we define its blow-up, which is a continuous mapping

$$
\pi \equiv \pi_{\mathcal{M}}: M \longrightarrow \mathcal{M}
$$


of a manifold $M$ with corners onto $\mathcal{M}$. [Recall that a manifold of dimension $n$ with corners is a Hausdorff space that is locally (i.e., in a neighborhood of each point) homeomorphic to the product $\overline{\mathbb{R}}_{+}^{k} \times \mathbb{R}^{n-k}, 0 \leq k \leq n$, where $k$ can depend on the point, and equipped with an atlas of such homeomorphisms with transition maps smooth up to the boundary.] We define the blow-up by the following "axioms":

1) If $\mathcal{M}$ is a stratified manifold of length 0 (i.e., a smooth manifold without boundary), then the blow-up has the form id $: \mathcal{M} \longrightarrow \mathcal{M}$ (i.e., $M=\mathcal{M}$ and the projection is the identity mapping).

2) If $\mathcal{N} \subset \mathcal{M}$ is an open (and hence stratified) submanifold, then we have the commutative diagram of blow-ups

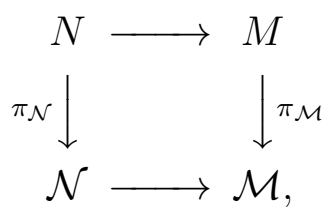

where the horizontal arrows are embeddings.

3) If $\mathcal{M}=\mathbb{R}^{m} \times K_{\Omega}$, where $\Omega$ is a stratified manifold, then the blow-up has the form

$$
\begin{aligned}
\pi_{\mathcal{M}}: \mathbb{R}^{m} \times \overline{\mathbb{R}}_{+} \times \widetilde{\Omega} & \longrightarrow \mathbb{R}^{m} \times K_{\Omega}, \\
(x, r, \widetilde{\omega}) & \longmapsto \begin{cases}(x, \mathbf{0}), & r=0 \\
\left(x, r, \pi_{\Omega}(\widetilde{\omega})\right), & r>0\end{cases}
\end{aligned}
$$

where $\pi_{\Omega}: \widetilde{\Omega} \longrightarrow \Omega$ is the blow-up of $\Omega$.

One can verify that these axioms by induction give a well-defined blow-up of every stratified manifold. Moreover, the blow-up is a diffeomorphism of the interior $M^{\circ}$ of the manifold $M$ onto the interior $\mathcal{M}^{\circ} \equiv \mathcal{M}_{0}^{\circ}$ of the manifold $\mathcal{M}$. In what follows, the term "blow-up" will be used for the mapping $\pi: M \longrightarrow \mathcal{M}$ as well as (by abuse of language) for the manifold $M$ with corners.

\subsection{The cone bundle}

Let $\mathcal{M}_{j}^{\circ}, j>0$, be an open stratum of $\mathcal{M}$. Formally extending the change of variables (4.2) written out for coordinate systems of type $j$ to arbitrarily large $r$, we see that over $\mathcal{M}_{j}^{\circ}$ there is a well-defined bundle with fiber $K_{\Omega}$, where $\Omega \equiv \Omega^{(j)}$ is a compact stratified manifold of length $j-1$.

Remark 23. It follows from the construction of the blow-up (we omit the corresponding computation for lack of space) that this bundle can be canonically (and smoothly) extended to the blow-up $M_{j}$ of the stratum $\mathcal{M}_{j}$. This remark will be useful in what follows, since the operator-valued symbols of our $\Psi$ DO will be defined over closed strata. 


\subsection{Measures on a stratified manifold}

On a compact stratified manifold $\mathcal{M}$ of length $k$, we introduce some natural metrics and the corresponding measures $\mu$, which give rise to spaces $L^{2}(\mathcal{M})=L^{2}(\mathcal{M}, \mu)$ of square integrable functions.

If a Riemannian metric is given, then we have a uniquely determined Riemannian volume element. Hence it suffices to describe the metrics. This will be done by induction over the stratification length.

1. On a compact stratified manifold of length 0 , we take an arbitrary Riemannian metric. The corresponding volume element is independent of the choice of the metric modulo equivalence (i.e., multiplication by an everywhere positive smooth function).

2. Suppose that we already know how to define metrics and measures on manifold of length $\leq k-1$. To do the same for manifolds of length $k$, it suffices to consider a neighborhood of the stratum $\mathcal{M}_{k}$ (since outside this neighborhood the stratification length is less than $k$ ). Moreover, it suffices to consider neighborhoods in which the cone bundle is trivialized. For such a neighborhood of the form $U \times K_{\Omega}$, where $U$ is a coordinate neighborhood on $\mathcal{M}_{k}$, we define the metric by the formula

$$
d s^{2}=d x^{2}+d r^{2}+r^{2} d \omega^{2},
$$

where $d \omega^{2}$ is the metric given by the induction assumption on $\Omega$. Globally, we obtain a metric on $\mathcal{M}$ by using a partition of unity.

Metrics obtained by this procedure are called metrics with edge degeneration.

Using formula (4.3), we write out an expression for the measure:

$$
d \mathrm{vol}=r^{n} d x d r d \operatorname{vol}_{\Omega},
$$

where $d \operatorname{vol}_{\Omega}$ is the volume element on $\Omega$, known by induction, and $n=\operatorname{dim} \Omega$ is the dimension of $\Omega$.

In what follows, all operators on $\mathcal{M}$ will be considered in the space

$$
L^{2}(\mathcal{M}) \equiv L^{2}(\mathcal{M}, d \operatorname{vol}),
$$

and all operators on the cone $K_{\Omega}$ will be considered in the space

$$
L^{2}\left(K_{\Omega}\right) \equiv L^{2}\left(K_{\Omega}, r^{n} d r d \operatorname{vol}_{\Omega}\right) .
$$

\subsection{The cotangent bundle}

By induction over the stratification length $k$, we define a space $_{\text {Vect }} \mathcal{M}$ of vector fields on the manifold $\mathcal{M}$.

1. For a smooth manifold $\mathcal{M}$, by $\operatorname{Vect}_{\mathcal{M}}$ we denote the space of all vector fields on $\mathcal{M}$.

2. For a manifold $\mathcal{M}$ of length $r$, we define $\operatorname{Vect}_{\mathcal{M}}$ as the $C^{\infty}(M)$-module that is locally (on the set $U \times \overline{\mathbb{R}}_{+} \times \widetilde{\Omega}$ corresponding to the coordinate neighborhood $U \times K_{\Omega}$ of $\mathcal{M})$ formed by the vector fields

$$
\theta=a \frac{\partial}{\partial v}+b \frac{\partial}{\partial r}+\frac{1}{r} \theta_{1}
$$

where $a$ and $b$ are smooth functions and $\theta_{1} \in$ Vect $_{\Omega}$.

The metric $d s^{2}$ defines a $C^{\infty}(M)$-valued pairing on Vect $_{\mathcal{M}}$. 
Proposition 24. The formula

$$
\langle\varphi(\theta), \mu\rangle=d s^{2}(\theta, \mu)
$$

specifies a bijection $\varphi$ of the space $\operatorname{Vect}_{\mathcal{M}}$ onto a $C^{\infty}(M)$-module $\Lambda^{1}(\mathcal{M}) \subset \Lambda^{1}(M)$ of differential forms on the blow-up $M$ of the manifold $\mathcal{M}$. The elements of $\Lambda^{1}(\mathcal{M})$ are exactly the forms than vanish on the fibers of the projection $p: M \longrightarrow \mathcal{M}$.

Proof. The assertion concerning the bijectivity follows from the fact that $\operatorname{Vect}_{M} \subset$ Vect $_{\mathcal{M}}$ and the embedding is epimorphic on the dense main stratum. The description of elements of $\Lambda^{1}(\mathcal{M})$ follows by a straightforward computation from (4.4).

Definition 25. The cotangent bundle $T^{*} \mathcal{M}$ of the manifold $\mathcal{M}$ is the (existing by the Swan theorem) bundle over the blow-up $M$ whose sections are elements of $\Lambda^{1}(\mathcal{M})$.

In a similar way, one defines the cotangent bundles of the strata $\mathcal{M}_{j}$.

\subsection{The space $C^{\infty}(\mathcal{M})$}

By induction on the length $k$ of a stratified manifold, we define a function space whose elements are called smooth functions on $\mathcal{M}$.

Definition 26. If $\mathcal{M}$ is a stratified manifold of length 0 , then the space $C^{\infty}(\mathcal{M})$ is defined in a standard way.

If $\mathcal{M}$ is a stratified manifold of length $k>0$, then a function $\varphi$ on $\mathcal{M}$ belongs to $C^{\infty}(\mathcal{M})$ if and only if

1) $\varphi \in C^{\infty}\left(\mathcal{M} \backslash \mathcal{M}_{k}\right)$;

2 ) in each coordinate neighborhood of type $k$ for sufficiently small $r$ the function $\varphi$ has the form

$$
\varphi(x, r, \omega)=\psi(x)
$$

where $\psi \in C^{\infty}\left(\mathbb{R}^{m}\right)$ is a smooth function.

It is easily seen that the space $C^{\infty}(\mathcal{M})$ is contained in the space $C(\mathcal{M})$ of continuous functions on $\mathcal{M}$ and is dense in it. Moreover, $\left[\mathcal{V}(\mathcal{M}), C^{\infty}(\mathcal{M})\right] \subset C^{\infty}(\mathcal{M})$.

\section{$5 \quad$ Pseudodifferential operators}

In this section, we describe the algebra of zero-order $\Psi \mathrm{DO}$ with parameters with smooth symbols on a stratified manifold $\mathcal{M}$. The definition of $\Psi D O$, as well as the definition of a stratified manifold, is based on induction over the stratification length. In contrast to the case of abstract operators considered in Sections 1-4, we shall assume that the parameter space $Y$ is a finite-dimensional vector space $V$ or, more generally, a vector bundle with fiber $V$ over a compact smooth manifold. To avoid cumbersome notation, we usually omit the variables pertaining to the base of the bundle. It is meant that the estimates written out below admit as many differentiations as desired along the base. 


\subsection{Negligible operators}

The calculus of $\Psi \mathrm{DO}$ with parameters will be constructed modulo some space of operator families the addition of which does not affect the Fredholm property and the index. Here we describe this space. Let $\mathcal{M}$ be a stratified manifold.

Definition 27. By $J_{\infty}(V, \mathcal{M}) \equiv J_{\infty}(\mathcal{M}) \equiv J_{\infty}$ we denote the space of smooth operator families

$$
D(v): L^{2}(\mathcal{M}) \longrightarrow L^{2}(\mathcal{M}), \quad v \in V,
$$

such that the following conditions are satisfied:

(i) all operators $D(v)$ are compact in $L^{2}(\mathcal{M})$;

(ii) the estimates

$$
\left\|\frac{\partial^{\beta} D(v)}{\partial v^{\beta}}\right\| \leq C_{\beta N}(1+|v|)^{-N}, \quad|\beta|, N=0,1,2, \ldots
$$

hold;

(iii) conditions (i) and (ii) remain valid if $D(v)$ is replaced by the product

$$
V_{1} \cdots V_{p} D(v) V_{p+1} \cdots V_{p+q}
$$

of arbitrary length $p+q, p, q \geq 0$, where $V_{1}, \ldots, V_{p+q} \in \mathcal{V}(\mathcal{M})$.

Proposition 28. The space $J_{\infty}$ is a two-sided $C^{\infty}(\mathcal{M})$-module. If $D \in J_{\infty}$ and $f_{\alpha}$ : $\mathcal{M} \longrightarrow \mathcal{M}$ is a family of diffeomorphisms smoothly depending on parameters $\alpha$, then the operator family $D_{\alpha} \equiv D_{\alpha}(v)=\left(f_{\alpha}^{*}\right)^{-1} D(v) f_{\alpha}^{*}$ is an element of $J_{\infty}$ smoothly depending on $\alpha$. (More precisely, the operator $D_{\alpha}(v)$ smoothly depends on $(\alpha, v)$, and conditions (i)-(iii) are satisfied locally uniformly with respect to $\alpha$ for the family $D(v)$ itself as well as for all its derivatives with respect to $\alpha$ ).

Proof. Obviously, conditions (i) and (ii) are preserved under right and left multiplication by $\varphi \in C^{\infty}(\mathcal{M})$. Condition (iii) is also preserved, since, as was already noted, $\left[\mathcal{V}(\mathcal{M}), C^{\infty}(\mathcal{M})\right] \subset C^{\infty}(\mathcal{M})$, so that it remains to use the Leibniz formula. Finally, let us prove the smoothness of the family $D_{\alpha}$. It depends on the parameter $\alpha$ strongly continuously, and the derivative $\partial D_{\alpha} / \partial \alpha_{j}$ has the form

$$
\frac{\partial D_{\alpha}}{\partial \alpha_{j}}=\left(f_{\alpha}^{*}\right)^{-1}[D(v), V] f_{\alpha}^{*}, \quad \text { where } V \in \mathcal{V}(\mathcal{M}),
$$

on a dense subset in $L^{2}(\mathcal{M})$. By condition (iii), the element $[D(v), V]$ belongs to $J_{\infty}$, so that, in particular, $\partial D_{\alpha} / \partial \alpha_{j}$ lies in $J_{\infty}$ and is uniformly bounded and strongly continuous. The same is true for higher-order derivatives, and hence the function $D_{\alpha}$ itself, as well as its derivatives, is continuous in the operator norm, so that the desired assertion follows readily. 


\subsection{Definition of pseudodifferential operators}

Let $\mathcal{M}$ be a compact stratified manifold of length $k$.

Definition 29. A pseudodifferential operator ( $\Psi D O)$ with parameter $v \in V$ on $\mathcal{M}$ is a smooth family of linear operators

$$
A(v): L^{2}(\mathcal{M}) \longrightarrow L^{2}(\mathcal{M})
$$

such that

(i) if $\varphi, \psi \in C^{\infty}(\mathcal{M})$ and $\operatorname{supp} \varphi \cap \operatorname{supp} \psi=\varnothing$, then $\psi A \varphi \in J_{\infty}$;

(ii) if $\varphi, \psi \in C^{\infty}(\mathcal{M})$ and the supports of both functions are contained in some coordinate neighborhood of type 0 , then the operator $\psi A \varphi$ is a classical zero-order $\Psi$ DO in $L^{2}$ with parameter $v$ in the sense of Agranovich-Vishik [1];

(iii) if $\varphi, \psi \in C^{\infty}(\mathcal{M})$ and the supports of both functions are contained in a coordinate neighborhood of type $l>0$, then the operator $\psi A \varphi$ is represented in the corresponding coordinate system $(x, r, \omega) \in \mathbb{R}^{m} \times K_{\Omega}$ in the form

$$
\psi A \varphi=P\left(\stackrel{3}{x}, \stackrel{3}{r}, \stackrel{3}{r} v,-i r \frac{\partial}{\partial x}, i r \frac{\partial}{\partial r}+i \frac{n+1}{2}\right)+R,
$$

where $R \in J_{\infty}, P(x, r, w, \eta, p)$ is a $\Psi$ DO with parameters $(w, \eta, p) \in \mathbb{R}^{\operatorname{dim} V+m+1}$ on $\Omega$ smoothly depending on the additional parameters $(x, r) \in \mathbb{R}^{m} \times \overline{\mathbb{R}}_{+}$and compactly supported with respect to these parameters, and quantization in the first term on the right-hand side in (5.4) is understood as follows:

$$
P\left(\stackrel{3}{x}, \stackrel{3}{r}, \stackrel{3}{r} v,-i r \frac{2}{\partial x}, i r \frac{\partial}{\partial r}+i \frac{n+1}{2}\right)=\widehat{P}\left(\stackrel{2}{x},-i \frac{1}{\partial x}\right)
$$

where the "operator-valued symbol" $\widehat{P}(x, \xi)$ is given by the formula

$$
\widehat{P}(x, \xi)=P\left(x, \stackrel{2}{r}, \stackrel{2}{r} v, \stackrel{2}{r} \xi, i r \frac{\partial}{\partial r}+i \frac{n+1}{2}\right),
$$

in which the function of the operator $i r \partial / \partial r$ is defined via the Mellin transform with the weight $\operatorname{line}^{6} \operatorname{Im} p=-(n+1) / 2$.

The set of $\Psi$ DO with a parameter $v \in V$ on $\mathcal{M}$ will be denoted by $\Psi \equiv \Psi(\mathcal{M}) \equiv \Psi(V, \mathcal{M})$, and by $J=\Psi \cap \mathcal{J}$ we denote its intersection with the ideal $\mathcal{J}$ of compact operators decaying as $v \rightarrow \infty$.

\footnotetext{
${ }^{6}$ This is consistent with the fact that the $\Psi$ DO $P$ is defined for real values of the parameter $p$.
} 


\subsection{The simplest properties of pseudodifferential operators}

In this subsection, we establish some elementary properties of $\Psi$ DO. In particular, we show that they form an algebra in which $J_{\infty}$ and $J$ are ideals; we also prove some estimates. We have not proved yet that condition (iii) in the definition of $\Psi$ DO is independent of the specific choice of a coordinate system (this will be established in the following subsection), but this does not affect our reasoning.

Theorem 30. The following assertions hold.

A. A $\Psi D O A \in \Psi(V, \mathcal{M})$ satisfies the estimates

$$
\left\|\frac{\partial^{\alpha} A(v)}{\partial v^{\alpha}}\right\| \leq C_{\alpha}(1+|v|)^{-|\alpha|}, \quad|\alpha|=0,1,2, \ldots,
$$

and $A^{(\alpha)} \in J$ for $|\alpha| \geq 1$.

B. The set $\Psi(\mathcal{M})$ is an algebra, where $C^{\infty}(\mathcal{M})$ is a subalgebra and $J$ and $J_{\infty}$ are ideals. Moreover,

$$
\left[C^{\infty}(\mathcal{M}), \Psi(\mathcal{M})\right] \subset J(\mathcal{M})
$$

C. If $V \in \mathcal{V}(\mathcal{M})$, then $[V, \Psi(\mathcal{M})] \subset \Psi(\mathcal{M})$.

Proof. We proceed by induction over the length $k$ of the manifold $\mathcal{M}$. For $k=0$, the assertion of the theorem is reduced to standard properties of $\Psi$ DO with a parameter on smooth manifolds, and it suffices to perform the induction step. It follows from property (i) that it suffices to verify everything for operators of the form $\psi A \varphi$ localized in some coordinate system. First, let us prove assertion A. It follows from assertion A known by the induction assumption for operators on manifolds of length less than $k$, in particular, from the estimates (5.7), that the operator family

$$
F(x, t, v, \xi, p)=P\left(x, e^{-t}, v e^{-t}, \xi e^{-t}, p\right)
$$

satisfies the estimates

$$
\begin{aligned}
& \left\|\frac{\partial^{|\alpha|+l+|\beta|+|\gamma|+k} F(x, t, v, \xi, p)}{\partial x^{\alpha} \partial t^{l} \partial v^{\beta} \partial \xi^{\gamma} \partial p^{k}}\right\| \leq C_{\alpha l \beta \gamma k}\left(e^{t}+|v|+|\xi|\right)^{-|\beta|-|\gamma|}(1+|p|)^{-k} \\
& \quad \leq \widetilde{C}_{\alpha l \beta \gamma k}(1+|v|+|\xi|)^{-|\beta|-|\gamma|}(1+|p|)^{-k}, \quad|\alpha|+l+|\beta|+|\gamma|+k=0,1,2, \ldots
\end{aligned}
$$

and has compact derivatives with respect to the parameters $(v, \xi, p)$. Now the fact that the operator on the right-hand side in (5.4) is well defined, is bounded, and satisfies condition $\mathrm{A}$ is a consequence of the following general assertion about $\Psi$ DO with operatorvalued symbols.

Proposition 31. (i) Let $H$ be a Hilbert space, and let

$$
H(x, \xi): H \longrightarrow H
$$


be a smooth symbol defined on $\mathbb{R}^{2 n}$, ranging in the set of bounded operators in $H$, and satisfying the estimates

$$
\left\|\frac{\partial^{\alpha+\beta} H(x, \xi)}{\partial x^{\alpha} \partial \xi^{\beta}}\right\| \leq C_{\alpha \beta}(1+|\xi|)^{-|\beta|}, \quad|\alpha|+|\beta|=0,1,2, \ldots .
$$

Then the operator

$$
\widehat{H} \equiv H\left(\stackrel{2}{x},-i \frac{\partial}{\partial x}\right): L^{2}\left(\mathbb{R}^{n}, H\right) \longrightarrow L^{2}\left(\mathbb{R}^{n}, H\right)
$$

is bounded, and its norm is bounded above by a constant that depends only on finitely many constants $C_{\alpha \beta}$. If, moreover, the symbol is compact-valued and decays as $(x, \xi) \rightarrow \infty$, then the operator (5.10) is compact.

(ii) If the symbol $H(x, \xi)$ is compactly supported with respect to $x$, then the assertion in (i) remains valid if $H(x, \xi)$ is smooth in $x$ and only measurable in $\xi$ and the estimates (5.9) are satisfied only for $|\beta|=0$. Under these conditions, if the symbol is measurable with respect to $\xi$ in the operator norm, is compact-valued, and uniformly decays as $|\xi| \rightarrow \infty$, then the operator (15.10) is compact.

Indeed, the change of variables $r=e^{-t}$ takes the cone $K_{\Omega}$ to the cylinder $\Omega \times \mathbb{R}$ and the operator $i r \partial / \partial r$ to $-i \partial / \partial t$. It remains to note that the operator $-i \partial / \partial t+i(n+1) / 2$ is self-adjoint in the space $L^{2}$ with weight $e^{-(n+1) t}$ on the cylinder, to which $L^{2}\left(K_{\Omega}\right)$ is taken by this change of variables, so that the substitution of this operator as an operator argument for $p$ is meaningful. It remains to apply Proposition 31 twice.

The proof of $\mathrm{B}$ and $\mathrm{C}$ is also by induction. It is based on the composition formula for operators with operator-valued symbol, given in the following proposition.

Proposition 32. Let $H_{j}(x, \xi), j=1,2$, be two symbols satisfying the estimates (5.9). Then

$$
\widehat{H}_{1} \widehat{H}_{2}=\widehat{H}
$$

where the symbol $H(x, \xi)$ also satisfies (5.9) and can be represented for each $N$ in the form

$$
H(x, \xi)=\sum_{|\gamma|=0}^{N-1} \frac{(-i)^{|\gamma|}}{|\gamma| !} \frac{\partial^{\gamma} H_{1}(x, \xi)}{\partial \xi^{\gamma}} \frac{\partial^{\gamma} H_{2}(x, \xi)}{\partial x^{\gamma}}+R_{N}(x, \xi)
$$

with a remainder $R_{N}(x, \xi)$ satisfying the estimates

$$
\left\|\frac{\partial^{\alpha+\beta} R_{N}(x, \xi)}{\partial x^{\alpha} \partial \xi^{\beta}}\right\| \leq C_{\alpha \beta}(1+|\xi|)^{-N-|\beta|}, \quad|\alpha|+|\beta|=0,1,2, \ldots
$$

If the $\xi$-derivatives of $H_{1}$ are compact-valued, then so is $R_{N}(x, \xi)$. If, moreover, at least one of the symbols $H_{j}$ decays as $x \rightarrow \infty$, then the operator $\widehat{R}_{N}$ is compact.

By applying this composition formula to all possible products arising in the verification of assertions $\mathrm{B}$ and $\mathrm{C}$, we arrive at the desired proof. 


\subsection{Invariance with respect to changes of variables}

In this subsection, we show that condition (iii) in Definition 29] is independent of the choice of a coordinate system and that $\Psi$ DO are invariant under changes of variables.

Theorem 33. The following assertions hold.

D. If condition (iii) in Definition [29 is satisfied for the operator $\psi A \varphi$ in some coordinate system, then it remains valid in any other coordinate system containing $\operatorname{supp} \psi \cup$ $\operatorname{supp} \varphi \cdot^{7}$

E. If $A \in \Psi(V, \mathcal{M})$ and $f_{\alpha}: \mathcal{M} \longrightarrow \mathcal{M}$ is a smooth family of diffeomorphisms, then

$$
A_{\alpha}=\left(f_{\alpha}^{*}\right)^{-1} A f_{\alpha}^{*}
$$

is a family of $\Psi D O$ with parameters $v \in V$ on $\mathcal{M}$ smoothly depending on the additional parameters $\alpha$.

Proof. The assertion of the theorem is known for $k=0$ (smooth manifolds). Assertion E follows from D (it suffices to consider a change of coordinates depending on a parameter), and so we shall prove only D. It suffices to consider the case in which both coordinate systems are of type $k$. (If both systems are of type less than $k$, then the desired result is known by induction assumption; if only one of the systems is of type $k$, then the support of the corresponding function $P$ is separated from $r=0$ in this system, so that the representative in this coordinate system can actually be assumed to be written out in a coordinate system of type less than $k$ ). Next, we can assume that the support of $P$ in both cases is contained in the domain $r<\varepsilon$, where $r$ is sufficiently small, so that the change of coordinates has the form

$$
x^{\prime}=f(x), \omega^{\prime}=g(x, \omega) .
$$

This transformation can be represented as the composition of two transformations, one of the form $x^{\prime}=f(x)$ and the other of the form $\omega^{\prime}=g(x, \omega)$. The transformation law for the function $P$ under these changes of variables can readily be written out. In the first case, we have

$$
P^{\prime}\left(x^{\prime}, r, r v, r \xi^{\prime}, p\right)=P(x, r, r v, r \xi, p),
$$

where $\left(x^{\prime}, \xi^{\prime}\right)=\left(f(x),\left(d f(x)^{*}\right)^{-1} \xi\right)$, and in the second case

$$
P^{\prime}(x, 0, r v, r \xi, p)=\left(g_{x}^{*}\right)^{-1} P(x, 0, r v, r \xi, p) g_{x}^{*}
$$

where $g_{x}=g(x, \cdot)$. (We omit the more complicated formula for $r>0$, which should be written out in coordinates on $\Omega$, since we do not need it.) The verification of both formulas is pretty similar to the finite-dimensional case.

\footnotetext{
${ }^{7}$ Formulas relating the $\Psi$ DO $P$ in the old and new coordinate systems will be given in the proof.
} 


\section{Symbols and quantization}

The ideal $J$ is contained in the ideal $\mathcal{J}$ of compact operators decaying as the parameter tends to infinity. Hence by Theorem 30, B $\Psi$ DO are local operators with a parameter; i.e., $\Psi(\mathcal{M}) \subset \mathcal{A}$. Thus we face the problem of computing the local operators $p_{z}(A) \in \mathcal{A}_{z}$, $z \in \mathcal{M}$, for a $\Psi$ DO $A \in \Psi(\mathcal{M})$.

\subsection{Infinitesimal operators and symbols of pseudodifferential operators}

On a stratified manifold, one can define local dilation groups in coordinates in a neighborhood of each point. Namely, let $z \in \mathcal{M}_{l}^{\circ}$ be some point. Consider a coordinate system of type $l$ centered at $z$. The point $z$ is depicted by the origin $(0, \mathbf{0})$ in $\mathbb{R}^{m} \times K_{\Omega}$, and we define the action of $\mathbb{R}_{+}$on $\mathbb{R}^{m} \times K_{\Omega}$ by the standard formula

$$
g_{\lambda}(x, r, \omega)=(\lambda x, \lambda r, \omega) .
$$

The one-parameter unitary group $U_{\lambda}$ associated with this action in the space $L^{2}\left(\mathbb{R}^{m} \times\right.$ $\left.K_{\Omega}, r^{n} d x d r d \operatorname{vol}_{\Omega}\right)=L^{2}\left(\mathbb{R}^{m}, L^{2}\left(K_{\Omega}, r^{n} d r d \operatorname{vol}_{\Omega}\right)\right), n=\operatorname{dim} \Omega$, is given by the formula

$$
\left[U_{\lambda} f\right](x, r, \omega)=\lambda^{(m+n+1) / 2} f(\lambda x, \lambda r, \omega),
$$

or

$$
\left[U_{\lambda} \mathbf{f}\right](x)=\lambda^{m / 2}\left[\varkappa_{\lambda} \mathbf{f}\right](\lambda x)
$$

where the function $\mathbf{f}(x)$ ranges in $\left.L^{2}\left(K_{\Omega}, r^{n} d r d \operatorname{vol}_{\Omega}\right)\right)$ and $\varkappa_{\lambda}$ is the one-parameter unitary group in the latter space given by the formula

$$
\left[\varkappa_{\lambda} u\right](r, \omega)=\lambda^{(n+1) / 2} u(\lambda r, \omega)
$$

We use this group to construct the infinitesimal operators of $\Psi \mathrm{DO}$.

Theorem 34. The following assertions hold.

F. Let $A \in \Psi(V, \mathcal{M})$ be a $\Psi D O$ with parameters on $\mathcal{M}$. Then for each $z \in \mathcal{M}_{l}^{\circ}$ and each coordinate system of type $l$ centered at $z$ the operator $A$ has an infinitesimal operator $i_{z}(A)$ invariant under $U_{\lambda}$. If $\varphi, \psi \in C^{\infty}(\mathcal{M})$ are functions supported in this coordinate neighborhood and equal to unity at $z$ and if $\psi A \varphi$ is represented in the local coordinates by formula (5.4), then the infinitesimal operator is given by

$$
i_{z}(A)=P\left(0,0, \stackrel{2}{r} v,-i r \frac{\partial}{\partial x}, i r \frac{\partial}{\partial r}+i \frac{n+1}{2}\right) .
$$

G. The infinitesimal operator commutes with translations along the stratum $\mathcal{M}_{l}^{\circ}$ (i.e., with respect to the variables $x$ ), and hence the symbol

$$
\sigma(A)(\xi)=P\left(0,0, \stackrel{2}{r} v, \stackrel{2}{r} \xi, i r \frac{\partial}{\partial r}+i \frac{n+1}{2}\right)
$$

is well defined. 
H. The symbol $\sigma(A)(\xi)$ smoothly depends on the point $x \in \mathcal{M}_{l}^{\circ}$ and is transformed under changes of variables as a function on the cotangent bundle of $\mathcal{M}_{l}^{\circ}$ ranging in the space of continuous operators in the spaces $L^{2}$ on the fibers of the cone bundle over $M_{l}$.

Proof. The proof of assertions $\mathrm{F}$ and $\mathrm{G}$ is by a straightforward computation with the use of Propositions 31 and 32 To prove H, we should additionally use formulas (5.15) and (5.16).

The symbol indicated in the theorem will be denoted by $\sigma_{l}(A)(x, \xi),(x, \xi) \in T_{0}^{*}\left(\mathcal{M}_{l}^{\circ}\right)$, and called the symbol of $A$ on the $l$ th stratum.

\subsection{Conditions for the Fredholm property of symbols}

By the general localization principle, the Fredholm property (with parameter) of a $\Psi \mathrm{DO}$ $A \in \Psi(V, \mathcal{M})$ is determined by the invertibility (for $\xi \neq 0$ ) of its symbols $\sigma_{l}(A)(x, \xi)$. Hence conditions ensuring the invertibility are of interest. Unfortunately, it is difficult to obtain such conditions in the general case, and so we shall study a weaker property, namely, the Fredholm property of the symbols. Consider the symbol (6.5).

Theorem 35. The symbol (6.5) is Fredholm for $\xi \neq 0$ if and only if

(i) all symbols $\sigma_{j}(P)(\widetilde{x}, \widetilde{\xi}), j=0, \ldots, l-1$, of the $\Psi D O P(0,0, w, \eta, p)$ with parameters on $\Omega$ are invertible for $\widetilde{\xi} \neq 0$;

(ii) the operator family $P(0,0,0,0, p)$ on $\Omega$ is invertible for all $p \in \mathbb{R}$.

Definition 36. The symbols $\sigma_{j}(P)$ will be called the symbols of the symbol $\sigma_{l}(A)$ and will be denoted by $\sigma_{j}\left(\sigma_{l}(A)\right), j=0, \ldots, l-1$. The family $P(0,0,0,0, p)$ will be called the conormal symbol of $\sigma_{l}(A)$ and will be denoted by $\sigma_{c}\left(\sigma_{l}(A)\right)$. It is easily seen that when symbols are multiplied, their symbols (and their conormal symbols) are also multiplied.

Proof of Theorem 35. It follows from Proposition 32 that if we multiply two symbols (6.5), then the corresponding $\Psi$ DO $P(0,0, w, \eta, p)$ are also multiplied modulo remainders that give compact-valued symbols. Hence the assertion of the theorem follows from the fact that $P\left(0,0, e^{-t} w, e^{-t} \xi, p\right)$ is compact-valued and decays as $|p| \rightarrow \infty$ and as $t \rightarrow-\infty$ if and only if all symbols of the family $P$ are zero; it decays also as $t \rightarrow \infty$ if and only if the conormal symbol of $P$ is zero.

The set of smooth symbols $\sigma(x, \xi)$ that have the form (6.5) for each $x$ will be denoted by $\Sigma\left(T_{0}^{*} \mathcal{M}_{l}\right)$.

\subsection{Compatibility conditions and quantization}

So far, we have only established that the symbols of a $\Psi$ DO $A \in \Psi(\mathcal{M})$ are defined on the interior of the corresponding cotangent bundles $T_{0}^{*} \mathcal{M}_{j}$. In fact, they can be extended by continuity up to the boundary (where the stratum is adjacent to lower-dimensional strata) and satisfy certain compatibility conditions on this boundary. 
Theorem 37. Let $A \in \Psi(\mathcal{M})$ be a $\Psi D O$ on $\mathcal{M}$. Then

K. each symbol $\sigma_{j}(A)(x, \xi)$ is a smooth function on $T_{0}^{*} \mathcal{M}_{j}$ up to the boundary;

L. at the points where a stratum $\mathcal{M}_{j}$ is adjacent to a stratum $\mathcal{M}_{l}, l>j$, the compatibility conditions

$$
\left.\sigma_{j}(D)\right|_{\mathcal{M}_{l}}=\sigma_{j}\left(\sigma_{l}(D)\right)
$$

are satisfied.

Proof. It suffices to compute the symbols of the operator (5.4) for $r=r_{0}>0$. They prove to be equal to the respective symbols of $P\left(x, r_{0}, w, \eta, p\right)$ and tend to the desired limits as $r_{0} \rightarrow 0$.

By $\Sigma(\mathcal{M}) \equiv \Sigma(V, \mathcal{M})$ we denote the subset of the direct product

$$
\prod_{j=0}^{k} \Sigma\left(T_{0}^{*} \mathcal{M}_{j}\right)
$$

formed by elements whose components pairwise satisfy the compatibility condition (6.6). Theorem [37 states that the tuple of symbols of a pseudodifferential operator $A \in \Psi(\mathcal{M})$ always lies in $\Sigma(\mathcal{M})$. It turns out that the converse is also true.

Theorem 38. Let $\left(\sigma_{0}, \ldots, \sigma_{k}\right) \in \Sigma(\mathcal{M})$. Then there exists a $\Psi D O A \in \Psi(\mathcal{M})$ such that

$$
\sigma_{j}(A)=\sigma_{j}, \quad j=0, \ldots, k .
$$

If the symbols $\sigma_{0}, \ldots, \sigma_{k}$ smoothly depend on parameters, then $A$ can also be assumed to depend smoothly on the same parameters.

Remark 39. The operator $A$ is uniquely determined modulo elements of the ideal $J$.

Proof. Let us prove this by induction.

1. For operators on a smooth manifold, the existence of an operator with a given symbol is known.

2. Now suppose that we need to construct an operator with given compatible symbols on a stratified manifold $\mathcal{M}$. By subtracting an operator of the form

$$
\phi(r) \sigma_{0}(D)(x,-i \partial / \partial x, v) \phi(r),
$$

where $\phi(r)$ is a cutoff function equal to zero for large $r$, we reduce the problem to the case in which the symbol corresponding to the stratum $\mathcal{M}_{k}$ is equal to zero identically, and so, by the compatibility conditions, all other symbols tend to zero when approaching this stratum.

It suffices to construct the corresponding operator on $\mathcal{M}$ locally, in a coordinate neighborhood $\mathbb{R}^{m} \times K_{\Omega}$ of type $k$. Take a family $\widetilde{P}(x, r, w, \eta, p)$ of $\Psi$ DO with parameters $(w, \eta, p)$ on $\Omega$ depending on the additional parameters $x, r$ such that $\sigma_{j}(P)=\sigma_{j}, j=0, \ldots, k-1$; this is possible by the induction assumption. Since all these symbols vanish for $r=0$, it follows that the family

$$
P(x, r, w, \eta, p)=\widetilde{P}(x, r, w, \eta, p)-\widetilde{P}(x, 0, w, \eta, p)
$$

has the same symbols $\sigma_{j}$ for $j \leq k-1$ and vanishes for $r=0$. By quantizing this family, we obtain the desired operator. The proof of the theorem is complete. 


\subsection{Main properties of pseudodifferential operators}

Theorem 40. The set $\Sigma\left(T^{*} X \times V\right)$, as well as the set $\Psi(\mathcal{M})$ of $\Psi D O$ on a stratified manifold $\mathcal{M}$, is a local $C^{*}$-algebra.

Proof. For the norm one can take the supremum of the operator norm over all parameter values. It has already been proved that these sets are algebras. Let us show that they are local $C^{*}$-algebras. To this end, we need to show that they are closed with respect to holomorphic functional calculus. Consider, say, the first of these algebras. If an element $D \in \Sigma\left(T^{*} X \times V\right)$ is invertible, then the inverse also lies in $\Sigma\left(T^{*} X \times V\right)$. This can readily be derived from the composition formula. Now let a function $f(z)$ be analytic in a neighborhood of the spectrum of an element $D$. Then

$$
f(D)=\frac{1}{2 \pi i} \oint_{\gamma} f(z)(z-D)^{-1} d z
$$

where $\gamma$ is a contour surrounding the spectrum and lying in the domain of analyticity of $f$. By the preceding, the operator $(z-D)^{-1}$ lies in $\Sigma\left(T^{*} X \times V\right)$. Moreover, one can readily show that this operator and its symbols continuously depend on the parameter $z$. Hence the operator $f(D)$ also lies in $\Sigma\left(T^{*} X \times V\right)$, and its symbols are given by the formula

$$
\sigma_{j}(f(D))=\frac{1}{2 \pi i} \oint_{\gamma} f(z)\left(z-\sigma_{j}(D)\right)^{-1} d z .
$$

Summarizing, we obtain the following theorem.

Theorem 41. The symbol mapping

$$
\begin{aligned}
\sigma: \Psi(V, \mathcal{M}) & \longrightarrow \bigoplus_{j=0}^{k} \Sigma\left(T^{*} \mathcal{M}_{j} \times V\right), \\
D & \longmapsto\left(\sigma_{0}(D), \ldots, \sigma_{k}(D)\right)
\end{aligned}
$$

is a homomorphism of local $C^{*}$-algebras and generates an isomorphism

$$
\sigma: \Psi(V, \mathcal{M}) / J \longrightarrow \Sigma(V, \mathcal{M}) \subset \bigoplus_{j=0}^{k} \Sigma\left(T^{*} \mathcal{M}_{j} \times V\right)
$$

onto the local $C^{*}$-algebra of symbols satisfying the compatibility conditions (6.6).

\subsection{Ellipticity and the Fredholm property}

Definition 42. An operator $D \in \Psi(V, \mathcal{M})$ is said to be elliptic if all symbols $\sigma_{j}(D)$, $j=0, \ldots, k$, are invertible outside the zero sections of the corresponding bundles.

The general localization principle implies the finiteness theorem in a standard way.

Theorem 43. Elliptic $\Psi D O$ on a compact stratified manifold $\mathcal{M}$ are Fredholm with a parameter. In particular, if $V \neq\{0\}$, then an operator elliptic with a parameter is invertible for large $|v|$. 


\section{Proof of Proposition 16}

Consider the composition

$$
B=\mathcal{F}_{t \rightarrow \xi} \widehat{B} \overline{\mathcal{F}}_{\xi \rightarrow t}
$$

We wish to show that $B$ can be represented as the pointwise action of an operatorvalued function $B(\xi)$ with the properties indicated in the proposition. Since the operator $\widehat{B}$ commutes with translations, it follows that $B$ commutes with the multiplications by the exponentials $\exp (i t \xi), t \in \mathbb{R}^{k}$, and hence, by continuity, with the multiplication by an arbitrary function $\varphi(\xi) \in C_{0}^{\infty}\left(\mathbb{R}^{k}\right)$. Now let $e \in H$ be an arbitrary vector, and let $\chi(\xi) \in C_{0}^{\infty}\left(\mathbb{R}^{k}\right)$ be an arbitrary function equal to unity on the support of $\varphi$. Then

$$
B(\varphi e)=B(\varphi \chi e)=\varphi B(\chi e) .
$$

The function $[B(\chi e)](\xi)$ belongs to $L^{2}\left(\mathbb{R}^{k}, H\right)$ and, in particular, is measurable. Relation (7.1) can be treated as equality a.e. of two measurable $H$-valued functions of $\xi$. At the points $\xi$ such that $\varphi(\xi) \neq 0$, one has a.e.

$$
[B(\chi e)](\xi)=\varphi^{-1}(\xi) B(\varphi e)(\xi),
$$

where the right-hand side is independent of $\chi$ and the left-hand side is independent of $\varphi$. Since the compactly supported functions $\varphi(\xi)$ and $\chi(\xi)$ are subjected to the only condition $\varphi \chi=\varphi$, we readily see that for each function $\varphi(\xi) \in C_{0}^{\infty}\left(\mathbb{R}^{k}\right)$ one has a.e.

$$
[B(\varphi e)](\xi)=\varphi(\xi) f(\xi),
$$

where $f(\xi)$ is a measurable $H$-valued function. A simple argument shows that

$$
\operatorname{ess} \sup \|f(\xi)\| \leq\|B\|=\|\widehat{B}\| \text {. }
$$

If $e=e_{1}+e_{2}$, then the corresponding functions $f(\xi)$ a.e. satisfy $f(\xi)=f_{1}(\xi)+f_{2}(\xi)$ (since this holds, by the linearity of the operator, for the functions $\varphi f$ ). Now let $\mathfrak{Z} \in H$ be a dense additive subgroup (say, the set of finite linear combinations of vectors of some basis with rational coefficients). For each $e \in \mathfrak{Z}$, we fix a representative of the corresponding element $f \in L^{2}\left(\mathbb{R}^{k}, H\right)$. Let us introduce the sets

$$
\Delta_{e}=\{\xi \mid\|f(\xi)\| \geq\|B\|\}, \quad \Delta_{e_{1} e_{2}}=\left\{\xi \mid f(\xi) \neq f_{1}(\xi)+f_{2}(\xi) \text {, where } e=e_{1}+e_{2}\right\} .
$$

They are of measure zero, and so is their union

$$
\Delta=\bigcup_{e \in \mathfrak{Z}} \Delta_{e} \cup \bigcup_{e_{1}, e_{2} \in \mathfrak{Z}} \Delta_{e_{1} e_{2}} .
$$

For each $\xi \in \mathbb{R}^{k} \backslash \Delta$, the mapping $e \mapsto f(\xi)$ defined on $\mathfrak{Z}$ is linear and is bounded by the norm $\|B\|$; hence its closure is a bounded linear operator $B(\xi): H \longrightarrow H$. Set $B(\xi)=0$ for $\xi \in \Delta$. The operator function this defined is measurable (since it is uniformly bounded and measurable on the dense set $\mathfrak{Z}$ ) and satisfies all desired conditions.

Finally, the continuous dependence of the operator $B(\xi)$ on the additional parameters is obvious. Indeed, by the preceding we have

$$
\left\|B_{\tau}(\xi)-B_{\tau_{0}}(\xi)\right\| \leq\left\|\widehat{B}_{y}-\widehat{B}_{y_{0}}\right\| \rightarrow 0
$$




\section{References}

[1] M. Agranovich and M. Vishik. Elliptic problems with parameter and parabolic problems of general type. Uspekhi Mat. Nauk, 19, No. 3, 1964, 53-161. English transl.: Russ. Math. Surv. 19 (1964), N 3, p. 53-157.

[2] A. Antonevich and A. Lebedev. Functional-Differential Equations. I. C ${ }^{*}$-Theory. Number 70 in Pitman Monographs and Surveys in Pure and Applied Mathematics. Longman, Harlow, 1994.

[3] W. Arveson. An Invitation to $C^{*}$-Algebras. Number 39 in Graduate Texts in Mathematics. Springer-Verlag, New York-Heidelberg-Berlin, 1976.

[4] M. F. Atiyah. Global theory of elliptic operators. In Proc. of the Int. Symposium on Functional Analysis, 1969, pages 21-30, Tokyo. University of Tokyo Press.

[5] D. Calvo, C.-I. Martin, and B.-W. Schulze. Symbolic structures on corner manifolds. In RIMS Conf. dedicated to L. Boutet de Monvel on Microlocal Analysis and Asymptotic Analysis, Kyoto, August 2004, 2005, pages 22-35, Tokyo. Keio University.

[6] J. Dauns and K.-H. Hofmann. Representation of Rings by Sections. Number 83 in Memoirs of the American Mathematical Society. American Mathematical Society, Providence, RI, 1968.

[7] J. Dixmier. Les $C^{*}$-algebres et leurs representations. Gauthier-Villars, Paris, 1969.

[8] Yu. Egorov and B.-W. Schulze. Pseudo-Differential Operators, Singularities, Applications. Birkhäuser, Boston, Basel, Berlin, 1997.

[9] I. Gohberg and N. Krupnik. One-Dimensional Linear Singular Integral Equations. I. Number 53 in Operator Theory: Advances and Applications. Birkhäuser, Basel, 1992.

[10] R. Melrose. Fibrations, compactifications and algebras of pseudodifferential operators. In L. Hörmander and A. Melin, editors, Partil Differential Equations and Mathematical Physics, 1996, pages 246-261. The Danish-Swedish Analysis Seminar, 1995, Birkhäuser.

[11] V. Nazaikinskii, A. Savin, B.-W. Schulze, and B. Sternin. Pseudodifferential operators on manifolds with singularities and localization. Russ. Acad. Sci. Doklady, 403, No. 6, 2005.

[12] V. Nistor. Pseudodifferential operators on non-compact manifolds and analysis on polyhedral domains. In Spectral geometry of manifolds with boundary and decomposition of manifolds, volume 366 of Contemp. Math., 2005, pages 307-328. Amer. Math. Soc., Providence, RI.

[13] B. A. Plamenevskij and V. N. Senichkin. On a class of pseudodifferential operators on $\mathbf{R}^{m}$ and on stratified manifolds. Sb. Math., 191, No. 5-6, 2000, 725-757. 
[14] B. A. Plamenevsky and V. N. Senichkin. Representations of an algebra of pseudodifferential operators with multidimensional discontinuities in the symbols. Math. USSR Izv., 31, No. 1, 1988, 143-169.

[15] B. A. Plamenevsky and V. N. Senichkin. Representations of $C^{*}$-algebras of pseudodifferential operators on piecewise-smooth manifolds. Algebra $i$ Analiz, 13, No. 6, 2001, 124-174.

[16] B.-W. Schulze. Pseudodifferential Operators on Manifolds with Singularities. NorthHolland, Amsterdam, 1991.

[17] I. B. Simonenko. A new general method of investigating linear operator equations of singular integral equation type. Izv. Akad. Nauk SSSR, Ser. Matem., 29, No. 3, 1965, 567-586, No. 4, 1965, 757-782.

[18] N. Vasilevski. Local principles in operator theory. In Lineinye operatory $v$ funktsionalnykh prostranstvakh. Tez. dokl. Severo-Kavkaz. reg. konf., Groznyi, 1989, pages $32-33$.

[19] N. Vasilevski. Convolution operators on standard CR-manifolds. II. Algebras of convolution operators on the Heisenberg group. Integr. Equat. Oper. Theory, 19, No. 3, 1994, 327-348.

[20] K. Yosida. Functional Analysis. Springer Verlag, Berlin, 1968. 